\title{
Jiří Neustupa
}

A contribution to the theory of stability of differential equations in Banach space

Czechoslovak Mathematical Journal, Vol. 29 (1979), No. 1, 27-52

Persistent URL: http://dml.cz/dmlcz/101576

\section{Terms of use:}

(C) Institute of Mathematics AS CR, 1979

Institute of Mathematics of the Czech Academy of Sciences provides access to digitized documents strictly for personal use. Each copy of any part of this document must contain these Terms of use.

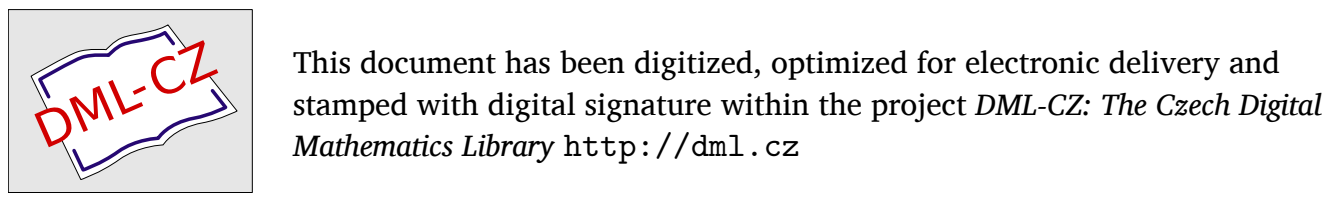




\section{A CONTRIBUTION TO THE THEORY OF STABILITY OF DIFFERENTIAL EQUATIONS IN BANACH SPACE}

JiŘí Neustupa, Praha

(Received December 15, 1976)

\section{INTRODUCTION}

In this paper, we shall investigate the uniform exponential stability and the uniform stability at constantly acting disturbances of the zero solution of abstract differential equations of the type

$$
\frac{\mathrm{d} U}{\mathrm{~d} t}=A U+B(t) U+N(t) U
$$

where $A$ and $B(t)$ are certain linear and $N(t)$ a certain nonlinear operator. In most cases the problem of stability of a solution $U_{0}$ of a generally nonlinear differential equation in a Banach space can be transformed by simple methods to the problem of stability of the zero solution of an equation of the type (0.1); that is why stability properties of the zero solution of equations of the type (0.1) are of a great importance.

From recent works about related mathematical problems we mention especially the paper of H. KIELHöFER [10], who treats the Lyapunov stability of the zero solution of a certain autonomous semilinear evolution equation in a Hilbert space and J. BARTÁK [2], who investigates the Lyapunov stability and the stability at constantly acting disturbances of solutions to abstract differential equations of the second order in a Hilbert space.

We list the assumed properties of the operators $A, B(t)$ and $N(t)$ in $\S 1$. Some auxiliary theorems are proved in this section, too. The uniform exponential stability and the stability at constantly acting disturbances of the zero solution of the equation $(0.1)$ are studied in $\S 2$ and $\S 3$. It is proved that these properties of the zero solution of the equation (0.1) are consequences of the uniform exponential stability of the so called linearized equation

$$
\frac{\mathrm{d} U}{\mathrm{~d} t}=A U+B(t) U
$$

In $\S 4$, it is shown that the derived results can be applied to the Navier-Stokes equations, the wave equation and the Timoshenko type equation. 
Let $X$ be a Banach space with the norm $\|\cdot\|_{0}$. The space of bounded linear operators from $X$ into $X$ will be denoted by $\mathscr{B}(X)$. If $I \subset E_{1}$ and $M \subset X$ then $\mathscr{C}^{n}(I ; M)$ (for $n=0,1, \ldots$ ) will be the set of $n$ times continuously differentiable mappings from $I$ into $M$ and $C^{n}(I ; M)($ for $n=1,2, \ldots)$ will be the Banach space of all elements $f$ from $\mathscr{C}^{n}(I ; M)$ with a finite norm

$$
\|f\|_{C^{n}(I ; M)}=\sum_{i=0}^{n} \sup _{t \in I}\left\|f^{(i)}(t)\right\|_{0} .
$$

Suppose that $A$ is a linear operator from $X$ into $X$ with the domain of definition $\mathscr{D}(A)$ such that $A$ is the generator of a semigroup of operators $\mathrm{e}^{A t}$ of the class $C_{0}$ in $X$, i.e. it holds

(i) $\lim _{t \rightarrow 0+}\left\|A x-\left(\mathrm{e}^{A t}-I\right) x / t\right\|_{0}=0$,

(ii) $\mathrm{e}^{A 0}=I$ and $\mathrm{e}^{A t} \in \mathscr{B}(X)$ for all $t \geqq 0$,

(iii) $\mathrm{e}^{A\left(t_{1}+t_{2}\right)}=\mathrm{e}^{A t_{1}} * \mathrm{e}^{A t_{2}}$ for all $t_{1}, t_{2} \geqq 0$,

(iv) $x$ being an arbitrary element of $X$, the mapping $t \mapsto \mathrm{e}^{A t} x$ belongs to $\mathscr{C}^{0}(\langle 0,+\infty) ; X)$.

Remark 1.1. The semigroup of the class $C_{0}$ is often called "the strongly continuous semigroup" in literature (see e.g. [3]).

It may be proved that $A$ is a closed operator, its domain of definition $\mathscr{D}(A)$ is dense in $X$, the limit $\lim _{t \rightarrow \infty}(1 / t) \log \left\|e^{A t}\right\|_{\mathscr{B}(X)}=\gamma$ exists and for each $\varrho>\gamma$ there exists $M_{\varrho} \in E_{1}$ such that the inequality

$$
\left\|\mathrm{e}^{A t}\right\|_{\mathscr{B}(X)} \leqq M_{\varrho} \cdot e^{\varrho t}
$$

holds for all $t \geqq 0$ (see [3]).

Let us denote

$$
\|x\|_{1}=\|x\|_{0}+\|A x\|_{0} \quad(\text { for } x \in \mathscr{D}(A))
$$

and let $X_{1}$ be the space $\mathscr{D}(A)$ with the norm $\|\cdot\|_{1}$. It follows from the closedness of the operator $A$ that $X_{1}$ is a Banach space.

Suppose that $B(t)$ (for $t \geqq 0$ ) is a linear operator from $X$ into $X$ with the domain of definition $\mathscr{D}(B)$ independent of $t$ and containing $\mathscr{D}(A)$. Let $\mathrm{e}^{A t} B(s) x \in X_{1}$ for all $x \in X_{1}, t \geqq 0, s \geqq 0$ and let the following assumption be fulfilled:

(v) there exists a function $k_{1}(t)$ so that $k_{1} \in L_{1}((0, l))$ for all $l>0$ and the inequality

$$
\left\|\mathrm{e}^{A t} B(s) x\right\|_{1} \leqq k_{1}(t)\|x\|_{1}
$$

holds for all $x \in \mathscr{D}(A), s \geqq 0$ and $t \geqq 0$. 
Let $\|\cdot\|$ and $\|\cdot\|_{0}$ be norms defined on $X_{1}$ and, respectively, on a subset $\mathscr{D}\left(\|\cdot\|_{0}\right)$ of $X$ containing $X_{1}$, so that there exist positive constants $c_{1}, c_{2}$ and $c_{3}$ such that

$$
\|x\|_{0} \leqq c_{1}\|x\|_{0} \leqq c_{2}\|x\| \leqq c_{3}\|x\|_{1} \quad\left(\text { for } x \in X_{1}\right) .
$$

Further, suppose that the following condition is satisfied:

(vi) there exists a function $k_{2}(t)$ and $p>1$ so that $k_{2} \in L_{p}((0, l))$ for all $l>0$ and

$$
\begin{gathered}
\left\|\mathrm{e}^{A t} x\right\| \leqq k_{2}(t)\|x\|_{0} \quad\left(\text { for all } t \geqq 0 \text { and } x \in \mathscr{D}\left(\|\cdot \cdot\| \|_{0}\right)\right), \\
\left.\left\|\mathrm{e}^{A t} B(s) x\right\| \mid \leqq k_{2}(t)\|x\| \| \quad \text { (for all } t \geqq 0, s \geqq 0 \text { and } x \in X_{1}\right) .
\end{gathered}
$$

Remark 1.2. We shall investigate the uniform exponential stability and the uniform stability at constantly acting disturbances with respect to the norm $\|\cdot\| \cdot \|$ in $\S 2$ and $\S 3$. The norm $\|\cdot\|_{0}$ will be used to determine the magnitude of disturbances in $\S 3$.

Let $N(t)$ be a generally nonlinear operator from $X$ into $X$ with the domain of definition $\mathscr{D}(N)$ independent of $t$ and containing $\mathscr{D}(A)$. Suppose that $\mathrm{e}^{A t} N(s) x \in X_{1}$ for all $x \in X_{1}, t \geqq 0, s \geqq 0$ and let

(vii) there exist $\alpha>0$ and functions $k_{3}(t, R), k_{4}(R)$ defined respectively on $\langle 0,+\infty) \times\langle 0,+\infty),\langle 0,+\infty)$ and such that $k_{3}(\cdot, R) \in L_{1}((0, l))$ for all $l>0$ and $R \geqq 0, k_{3}(t,$.$) and k_{4}$ are nondecreasing functions of $R$ for all $t \geqq 0$ and the inequalities

$$
\left\|\mathrm{e}^{A t} N(s) x\right\| \leqq k_{3}(t, R)\|x\|\left\|^{1+\alpha}, \quad\right\| N(s) x\left\|_{0} \leqq k_{4}(R)\right\| x\|\|^{\mathbf{1}+\alpha}
$$

hold for all $s \geqq 0, t \geqq 0$ and $x \in X_{1}$ such that $\|x\| \leqq R$.

Further, we shall study differential equations of the type (0.1). By solutions of these equations on an interval $I \subset\langle 0,+\infty)$ we shall understand only functions from $\mathscr{C}^{0}\left(I ; X_{1}\right) \cap \mathscr{C}^{1}(I ; X)$ which satisfy the given equation on $I$. In the case of solutions on $\langle 0,+\infty)$ we shall not mention this interval explicitly.

Lemma 1.1. Let $\tau \geqq 0$. Then $U$ is a solution of the equation $(0.2)$ on $\langle\tau,+\infty)$ if and only if $U \in \mathscr{C}^{0}\left(\langle\tau,+\infty) ; X_{1}\right) \cap \mathscr{C}^{1}(\langle\tau,+\infty) ; X)$ and

$$
U(t)=\mathrm{e}^{A(t-\tau)} U(\tau)+\int_{\tau}^{t} \mathrm{e}^{A(t-\sigma)} B(\sigma) U(\sigma) \mathrm{d} \sigma \quad \text { for } \quad t \geqq \tau .
$$

Proof. If $U$ is a solution of $(0.2)$ on $\langle\tau,+\infty)$ then $U \in \mathscr{C}^{0}\left(\langle\tau,+\infty) ; X_{1}\right) \cap$ $\cap \mathscr{C}^{1}(\langle\tau,+\infty) ; X)$ and

$$
\mathrm{e}^{A(t-\sigma)} \frac{\mathrm{d} U(\sigma)}{\mathrm{d} \sigma}=\mathrm{e}^{A(t-\sigma)} A U(\sigma)+\mathrm{e}^{A(t-\sigma)} B(\sigma) U(\sigma)
$$

for all $t \geqq \tau$ and $\sigma \in\langle\tau, t\rangle$. Thus,

$$
\int_{\tau}^{t} \mathrm{e}^{A(t-\sigma)} \frac{\mathrm{d} U(\sigma)}{\mathrm{d} \sigma} \mathrm{d} \sigma=\int_{\tau}^{t} \mathrm{e}^{A(t-\sigma)} A U(\sigma) \mathrm{d} \sigma+\int_{\tau}^{t} \mathrm{e}^{A(t-\sigma)} B(\sigma) U(\sigma) \mathrm{d} \sigma .
$$


All integrals in (1.7) converge in $X$ as follows from the estimates

$$
\begin{gathered}
\left\|\mathrm{e}^{A(t-\sigma)} \frac{\mathrm{d} U(\sigma)}{\mathrm{d} \sigma}\right\|_{0} \leqq M_{\varrho} \mathrm{e}^{\varrho(t-\sigma)}\left\|\frac{\mathrm{d} U(\sigma)}{\mathrm{d} \sigma}\right\|_{0} \leqq M_{\varrho} \mathrm{e}^{\varrho(t-\sigma)}\|U\|_{C^{1}(\langle\tau, t\rangle ; X)}, \\
\left\|\mathrm{e}^{A(t-\sigma)} A U(\sigma)\right\|_{0} \leqq M_{\varrho} \max \left(1, \mathrm{e}^{\varrho(t-\tau)}\right)\|U\|_{C^{0}\left(\langle\tau, t\rangle ; X_{1}\right)}, \\
\left\|\mathrm{e}^{A(t-\sigma)} B(\sigma) U(\sigma)\right\|_{0} \leqq k_{1}(t-\sigma)\|U\|_{C^{0}\left(\langle\tau, t\rangle ; X_{1}\right)} .
\end{gathered}
$$

Using the integration by parts on the left hand side of the equation (1.7), we get (1.6).

Conversely, let $U \in \mathscr{C}^{0}\left(\langle\tau,+\infty) ; X_{1}\right) \cap \mathscr{C}^{1}(\langle\tau,+\infty) ; X)$ and let $U$ satisfy (1.6). It follows from the estimate

$$
\left\|A \mathrm{e}^{A(t-\sigma)} B(\sigma) U(\sigma)\right\|_{0} \leqq\left\|\mathrm{e}^{A(t-\sigma)} B(\sigma) U(\sigma)\right\|_{1} \leqq k_{1}(t-\sigma)\|U\|_{C^{0}\left(\langle\tau, t\rangle ; X_{1}\right)}
$$

that the integrals

$$
\int_{\tau}^{t} \frac{\mathrm{d}}{\mathrm{d} t} \mathrm{e}^{A(t-\sigma)} B(\sigma) U(\sigma) \mathrm{d} \sigma, \quad \int_{\tau}^{t} A \mathrm{e}^{A(t-\sigma)} B(\sigma) U(\sigma) \mathrm{d} \sigma
$$

converge, hence we can interchange the order of integration, differentiation with respect to $t$ or application of the operator $A$ in these integrals after substituting $U$ from (1.6) into (0.2). Then after an easy computation we can verify that $U$ satisfies the equation $(0.2)$.

Lemma 1.2. Let $\tau \geqq 0$ and let $U$ be a solution of the equation (0.1) on the interval $\langle\tau,+\infty)$. Then $U$ satisfies the relation

$$
\begin{aligned}
U(t) & =\mathrm{e}^{A(t-\tau)} U(\tau)+\int_{\tau}^{t} \mathrm{e}^{A(t-\sigma)} B(\sigma) U(\sigma) \mathrm{d} \sigma+ \\
& +\int_{\tau}^{t} \mathrm{e}^{A(t-\sigma)} N(\sigma) U(\sigma) \mathrm{d} \sigma \quad(\text { for } t \geqq \tau) .
\end{aligned}
$$

Proof. The equality (1.8) can be derived from the equation (0.1) in the same way as the equality (1.6) from the equation (0.2). It is only the last integral on the right hand side of (1.8) which is added here. The convergence of this integral in $X$ follows from the estimates

$$
\begin{gathered}
\left\|e^{A(t-\sigma)} N(\sigma) U(\sigma)\right\|_{0} \leqq c_{2}\left\|\mathrm{e}^{A(t-\sigma)} N(\sigma) U(\sigma)\right\| \leqq \\
\leqq c_{2} k_{3}(t-\sigma,\|U(\sigma)\|) \cdot\|U\|^{1+\alpha} \leqq c_{2}\left(\frac{c_{3}}{c_{2}}\right)^{1+\alpha} k_{3}\left(t-\sigma, \frac{c_{3}}{c_{2}}\|U(\sigma)\|_{1}\right) \cdot\|U(\sigma)\|_{1}^{1+\alpha} \leqq \\
\leqq c_{2}\left(\frac{c_{3}}{c_{2}}\right)^{1+\alpha} k_{3}\left(t-\sigma, \frac{c_{3}}{c_{2}}\|U\|_{C^{0}\left(\langle\tau, t\rangle ; X_{1}\right)}\right)\|U\|_{C^{0}\left(\langle\tau, t\rangle ; X_{1}\right)} .
\end{gathered}
$$


Lemma 1.3. Let $\tau \geqq 0$ and $x_{0} \in X_{1}$. Then there exists a unique solution $U$ of the equation $(0.2)$ on $\langle\tau,+\infty)$ satisfying the initial condition $U(\tau)=x_{0}$. This solution depends continuously on the initial value $x_{0}$ in the sense that

$$
\begin{gathered}
\|U(t)\|_{1} \leqq M_{\rho}\left\{\max \left(1, e^{\varrho t}\right)+\right. \\
\left.+\int_{\tau}^{t} \max \left(1, \mathrm{e}^{\varrho \sigma}\right) k_{1}(t-\sigma) \exp \left[\int_{\tau}^{\sigma} k_{1}(t-\vartheta) \mathrm{d} \vartheta\right] \mathrm{d} \sigma\right\}\left\|x_{0}\right\|_{1} .
\end{gathered}
$$

Proof. According to Lemma 1.1, we may deal with the integro-differential equation

$$
U(t)=\mathrm{e}^{A(t-\tau)} x_{0}+\int_{\tau}^{t} \mathrm{e}^{A(t-\sigma)} B(\sigma) U(\sigma) \mathrm{d} \sigma
$$

instead of the equation (0.2). Denoting

$$
G(U)(t)=\int_{\tau}^{t} \mathrm{e}^{A(t-\sigma)} B(\sigma) U(\sigma) \mathrm{d} \sigma, G^{0}(U)(t)=U(t)
$$

and regarding the linearity of the operator $G$, we may transform the equation (1.10). into the form

$$
U(t)=\sum_{k=0}^{n} G^{k}(V)(t)+G^{n+1}(U)(t)
$$

where $V(\sigma)=\mathrm{e}^{A(\sigma-\tau)} x_{0}$ and $n$ is an arbitrary natural number. If $T>0$ then

$$
\begin{gathered}
\|G(U)\|_{C^{0}\left(\langle\tau, \tau+T\rangle ; X_{1}\right)} \leqq \max _{t \in\langle\tau, \tau+T\rangle} \int_{\tau}^{t} k_{1}(t-\sigma)\|U(\sigma)\|_{1} \mathrm{~d} \sigma \leqq \\
\leqq\left(\int_{\tau}^{\tau+T} k_{1}(\tau+T-\sigma) \mathrm{d} \sigma\right) \cdot\|U\|_{C^{0}\left(\langle\tau, \tau+T\rangle ; X_{1}\right)},
\end{gathered}
$$

and it means that

$$
\|G\|_{\mathscr{B}\left(C^{0}\left(\langle\tau, \tau+T\rangle ; X_{1}\right)\right)} \leqq \int_{\tau}^{\tau+T} k_{1}(\tau+T-\sigma) \mathrm{d} \sigma=\int_{0}^{T} k_{1}(\sigma) \mathrm{d} \sigma .
$$

Choosing $T>0$ sufficiently small, we may achieve that $\| G_{\mathscr{B}\left(C^{\circ}\left(\langle\tau, \tau+T\rangle ; X_{1}\right)\right)} \leqq h<1$. Then the series

$$
\sum_{k=0}^{+\infty} G^{k}(V)(t) \quad\left(\text { with } V(\sigma)=e^{A(\sigma-\tau)} x_{0}\right)
$$

converges in the norm of the space $C^{0}\left(\langle\tau, \tau+T\rangle ; X_{1}\right)$. Similarly, we can prove also the convergence of the series (1.12) in the norm of the space $C^{1}(\langle\tau, \tau+T\rangle ; X)$. Hence the sum of the series (1.12) (we shall denote it $S(t, \tau) x_{0}$ ) belongs to 
$C^{0}\left(\langle\tau, \tau+T\rangle ; X_{1}\right) \cap C^{1}(\langle\tau, \tau+T\rangle ; X)$. It follows from (1.11) that $S(t, \tau) x_{0}$ satisfies the equation (1.10), i.e. $S(t, \tau) x_{0}$ is also the solution of $(0.2)$ on $\langle\tau, \tau+T\rangle$. It may be easily shown that

$$
S\left(t_{1}, \tau\right)=S\left(t_{1}, t_{2}\right) * S\left(t_{2}, \tau\right) \quad\left(\text { for } \tau \leqq t_{2} \leqq t_{1} \leqq \tau+T\right) .
$$

Using this equality, we can extend the definition of the operator $S(t \tau)$ for all $t \geqq \tau$ in the following way: Every number $t \geqq \tau$ can be expressed in the form $t=\tau+$ $+m T+t^{\prime}$, where $m$ is a natural number and $t^{\prime} \in\langle 0, T)$. Then we define

$$
S(t, \tau)=S(t, \tau+m T) * S(\tau+m T, \tau+(m-1) T) * \ldots * S(\tau+T, \tau) .
$$

The function $U(t)=S(t, \tau) x_{0}$ is the solution of the equation $(0.2)$ on $\langle\tau,+\infty)$ and $U(\tau)=x_{0}$.

Returning now to (1.10), we can obtain the estimate

$$
\|U(t)\|_{1} \leqq M_{\varrho} \cdot \max \left(1, \mathrm{e}^{e t}\right)\left\|x_{0}\right\|_{1}+\int_{\tau}^{t} k_{1}(t-\sigma)\|U(\sigma)\|_{1} \mathrm{~d} \sigma
$$

and applying the so called generalized Bellman inequality (see e.g. [4], p. 78), we get (1.9). The uniqueness of the solution $U$ is a consequence of the inequality (1.9).

\section{§2. THE UNIFORM EXPONENTIAL STABILITY}

Definition 2.1. The zero solution of the equation (0.1) is uniformly exponentially stable with respect to the norm $\|\cdot\| \cdot \|$ if there exist positive numbers $\delta, K_{1}$ and $K_{2}$ so that the implication

$$
\|U(\tau)\| \mid<\delta \Rightarrow\|U(t)\| \leqq K_{1}\|U(0)\| \| \mathrm{e}^{-K_{2}(t-\tau)}
$$

is valid for each $\tau \geqq 0$, for every solution $U$ of $(0.1)$ on the interval $\langle\tau, \tau+L)$ (where $L \in(0,+\infty\rangle$ depends on $U$ ) and for all $t \in\langle\tau, \tau+L$ ).

Throughout Sections 2 and 3 we shall deal with the stability with respect to the norm $\|\cdot|\||$ only, without repeating it any more.

We do not study the existence of solutions of the equation (0.1) in this work. Nevertheless, it is known that some solutions of $(0.1)$ with initial values sufficiently small exist in many special cases of the equation (0.1), including important equations of mathematical physics. Otherwise, of course, the investigation of the uniform exponential stability of the zero solution of $(0.1)$ would have no sense.

The main result of this section is the following theorem:

Theorem 2.1. Let the zero solution of the equation (0.2) be uniformly exponentially stable. Then the zero solution of the equation (0.1) has the same property. 
Proof. In virtue of the linearity of the equation (0.2), the uniform exponential stability of the zero solution of this equation means the existence of positive constants $K_{1}$ and $K_{2}$ such that the inequality

$$
\|V(t)\| \leqq K_{1}\|V(\tau)\| \mathrm{e}^{-K_{2}(t-\tau)}
$$

holds for every solution $V$ of the equation $(0.2)$ and all $\tau \geqq 0, t \geqq \tau$.

Suppose that $\lambda$ is an arbitrary number from the interval $\left(0, K_{2}\right)$. It may be easily verified that $U$ solves (0.1) if and only if the function $\bar{U}(t)=\mathrm{e}^{\lambda t} U(t)$ solves the equation

$$
\frac{\mathrm{d} \bar{U}}{\mathrm{~d} t}=A \bar{U}+B(t) \bar{U}+\lambda \bar{U}+\mathrm{e}^{\lambda t} N(t)\left(\mathrm{e}^{-\lambda t} \bar{U}\right)
$$

Similarly, $V$ is a solution of $(0.2)$ if and only if $\bar{V}(t)=\mathrm{e}^{\lambda t} V(t)$ is a solution of the equation

$$
\frac{\mathrm{d} \bar{V}}{\mathrm{~d} t}=A \bar{V}+B(t) \bar{V}+\lambda \bar{V}
$$

It follows from (2.2) that if $\bar{V}$ is a solution of (2.4) and $\tau \geqq 0, t \geqq \tau$ then

$$
\|\bar{V}(t)\|\left|\leqq K_{1}\|\bar{V}(\tau)\|\right| \mathrm{e}^{\left(\lambda-K_{2}\right)(t-\tau)} .
$$

Further, let $\tau$ be any nonnegative number and $\bar{U}$ a solution of the equation (2.3) on $\langle\tau, \tau+L)$. We shall prove that, if

$$
\|\bar{U}(\tau)\|<\frac{\delta}{2 K_{1}} \mathrm{e}^{\lambda \tau}
$$

and $\delta$ is sufficiently small, then

$$
\|\bar{U}(t)\|<2 K_{1} \mid\|\bar{U}(\tau)\| \quad(\text { for } t \in\langle\tau, \tau+L)) .
$$

Let us suppose that this is not true, i.e. that there exists $t_{0}>\tau$ such that

$$
\begin{gathered}
\|\| \bar{U}\left(t_{0}\right)\|\|=2 K_{1}\|\bar{U}(\tau)\|, \\
\|\bar{U}(t)\|<\left\|\bar{U}\left(t_{0}\right)\right\| \| \quad\left(\text { for } t \in\left\langle\tau, t_{0}\right)\right) .
\end{gathered}
$$

By Lemma 1.3 there exists a solution $\bar{V}$ of $(2.4)$ on $\langle\tau,+\infty)$ such that $\bar{V}(\tau)=\bar{U}(\tau)$. Firstly, suppose that $t_{0} \in(\tau, \tau+2 r)$, where $r=\left(1 /\left(K_{2}-\lambda\right)\right) \log K_{1}\left(\right.$ i.e. $\mathrm{e}^{\left(K_{2}-\lambda\right) r}=$ $\left.=K_{1}\right)$. We shall use the following lemma:

Lemma 2.1. Let $T \geqq 0$ and let $\tilde{U}, \tilde{V}$ be solutions of the equations (2.3), (2.4), respectively, on $\langle T, T+L)$ so that $\tilde{U}(T)=\tilde{V}(T)$. Then

$$
\|\tilde{U}(t)-\tilde{V}(t)\| \mid \leqq Z(t-T) \int_{T}^{t} k_{3}\left(t-\sigma, \mathrm{e}^{-\lambda \sigma}\|\tilde{U}(\sigma)\|\right) \mathrm{e}^{-\lambda \alpha \sigma}\|\tilde{U}(\sigma)\| \|^{1+\alpha} \mathrm{d} \sigma
$$


for $t \in\langle T, T+L)$, where $Z$ is a certain real positive nondecreasing function on the interval $\langle 0,+\infty)$.

The proof of this lemma will be given after completing the proof of Theorem 2.1. An explicit form of the function $Z$ will be derived as well.

Making use of the estimate (2.10) (where we put $\tilde{U}=\bar{U}, \tilde{V}=\bar{V}, T=\tau$ ), we obtain

$$
\begin{gathered}
\left\|\bar{U}\left(t_{0}\right)\right\| \leqq\left\|\bar{V}\left(t_{0}\right)\right\|\left\|+Z\left(t_{0}-\tau\right) \int_{\tau}^{t_{0}} k_{3}\left(t_{0}-\sigma, \mathrm{e}^{-\lambda \sigma}\|\bar{U}(\sigma)\|\right) \mathrm{e}^{-\lambda \alpha \sigma}\right\| \bar{U}(\sigma)\|\|^{1+\alpha} \mathrm{d} \sigma \leqq \\
\leqq K_{1}\|\bar{V}(\tau)\| \| \mathrm{e}^{\left(\lambda-K_{2}\right)\left(t_{0}-\tau\right)}+ \\
+Z(2 r) \int_{\tau}^{t_{0}} k_{3}\left(t_{0}-\sigma, 2 K_{1} \mathrm{e}^{-\lambda \sigma}\|\bar{U}(\tau)\|\right) \mathrm{e}^{-\lambda \alpha \sigma}\left(2 K_{1}\|\bar{U}(\tau)\|\right)^{1+\alpha} \mathrm{d} \sigma \leqq \\
\leqq K_{1}\|\bar{U}(\tau)\|+Z(2 r) \mathrm{e}^{-\lambda \alpha \tau}\left(2 K_{1}\|\bar{U}(\tau)\| \mid\right)^{1+\alpha} \int_{\tau}^{\tau+2 r} k_{3}\left(\tau+2 r-\sigma, \delta \mathrm{e}^{-\lambda(\sigma-\tau)}\right) \mathrm{d} \sigma .
\end{gathered}
$$

Thus, we have

$$
\begin{gathered}
\left\|\bar{U}\left(t_{0}\right)\right\|=2 K_{1}\|\bar{U}(\tau)\| \leqq K_{1}\|\bar{U}(\tau)\|+ \\
+\left(2 K_{1}\|\bar{U}(\tau)\|\right)^{1+\alpha} \mathrm{e}^{-\lambda \alpha \tau} Z(2 r) \int_{\tau}^{\tau+2 r} k_{3}(\tau+2 r-\sigma, \delta) \mathrm{d} \sigma
\end{gathered}
$$

and using (2.6), we get

$$
1 \leqq 2 \delta^{\alpha} Z(2 r) \int_{0}^{2 r} k_{3}(\sigma, \delta) \mathrm{d} \sigma .
$$

Secondly, let $t_{0} \geqq \tau+2 r$. Then $t_{0}$ can be expressed in the form $t_{0}=\tau+r n+l$, where $n$ is a natural number and $l \in\langle r, 2 r)$. Let $\bar{V}_{n}$ be a solution of the linear equation (2.4) on $\langle\tau+r n,+\infty)$ such that $\bar{V}_{n}(\tau+r n)=\bar{U}(\tau+r n)$ (the existence of such a solution $\bar{V}_{n}$ is ensured by Lemma 1.3). Then

$$
\left\|\overline { V } _ { n } ( t ) \left|\left\|\leqq K_{1} \mid\right\| \bar{U}(\tau+r n) \| \mathrm{e}^{\left(\lambda-K_{2}\right)(t-\tau-r n)} \quad(\text { for } t \geqq \tau+r n)\right.\right.
$$

(see (2.5)). Now, writing $\tilde{U}=\bar{U}, \tilde{V}=\bar{V}$ and $T=\tau+r n$ in (2.10), we get the inequality

$$
\begin{gathered}
\left\|\bar{U}\left(t_{0}\right)\right\|\|\leqq\| \bar{V}_{n}\left(t_{0}\right) \|+ \\
+\left.Z\left(t_{0}-\tau-r n\right) \int_{\tau+r n}^{t_{0}} k_{3}\left(t_{0}-\sigma, \mathrm{e}^{-\lambda \sigma}\|\bar{U}(\sigma)\| \|\right) \mathrm{e}^{-\lambda \alpha \sigma}\|\bar{U}(\sigma)\|\right|^{1+\alpha} \mathrm{d} \sigma
\end{gathered}
$$

and in virtue of (2.12), we have

$$
\begin{aligned}
& \left\|\left|\bar{U}\left(t_{0}\right)\left\|\mid \leqq K_{\mathrm{i}}\right\| \bar{U}(\tau+r n)\|\| \mathrm{e}^{\left(\lambda-K_{2}\right)\left(t_{0}-\tau-r n\right)} .\right.\right. \\
& . Z(2 r) \int_{\tau+r n}^{t_{0}} k_{3}\left(t_{0}-\sigma, \delta\right) \mathrm{e}^{-\lambda \alpha \sigma}\|\bar{U}(\sigma)\| \|^{1+\alpha} \mathrm{d} \sigma .
\end{aligned}
$$


Since $r \leqq l$ and $K_{1} \mathrm{e}^{\left(\lambda-K_{2}\right) r}=1$, one has

$$
\begin{gathered}
\left\|\bar{U}\left(t_{0}\right)\right\| \leqq\|\bar{U}(\tau+r n)\|+ \\
+Z(2 r) \int_{\tau+r n}^{t_{0}} k_{3}\left(t_{0}-\sigma, \delta\right) \mathrm{e}^{-\lambda \alpha \sigma}\|\bar{U}(\sigma)\|^{1+\alpha} \mathrm{d} \sigma .
\end{gathered}
$$

Similarly, it can be proved that

$$
\begin{gathered}
\|\bar{U}(\tau+r j)\| \leqq\|\bar{U}(\tau+r(j-1))\|+ \\
+Z(2 r) \int_{\tau+r(j-1)}^{\tau+r j} k_{3}\left(t_{0}-\sigma, \delta\right) \mathrm{e}^{-\lambda \alpha \sigma}\|\bar{U}(\sigma)\| \|^{1+\alpha} \mathrm{d} \sigma
\end{gathered}
$$

for $j=1,2, \ldots, n$. Thus, using (2.13) and (2.14), we get

$$
\begin{gathered}
\left\|\bar{U}\left(t_{0}\right)\right\| \leqq\|\bar{U}(\tau)\|+\left(2 K_{1}\|\bar{U}(\tau)\|\right)^{1+\alpha} . \\
. Z(2 r)\left[\sum_{j=1}^{n} \int_{\tau+r(j-1)}^{\tau+r j} k_{3}(\tau+r j-\sigma, \delta) \mathrm{e}^{-\lambda \alpha \sigma} \mathrm{d} \sigma+\int_{\tau+r n}^{t_{0}} k_{3}\left(t_{0}-\sigma, \delta\right) \mathrm{e}^{-\lambda \alpha \sigma} \mathrm{d} \sigma\right] \leqq \\
\leqq\|\bar{U}(\tau)\|+\left(2 K_{1}\|\bar{U}(\tau)\|\right)^{1+\alpha} Z(2 r)\left[\int_{0}^{2 r} k_{3}(\sigma, \delta) \mathrm{d} \sigma\right] \sum_{j=0}^{n} \mathrm{e}^{-\lambda \alpha(\tau+r j)} .
\end{gathered}
$$

Hence it is

$2 K_{1}\|\bar{U}(\tau)\|=\left\|\bar{U}\left(t_{0}\right)\right\||\leqq\|\bar{U}(\tau)\||+\left(2 K_{1}\|\bar{U}(\tau)\|\right)^{1+\alpha} Z(2 r)\left[\int_{0}^{2 r} k_{3}(\sigma, \delta) \mathrm{d} \sigma\right] \frac{\mathrm{e}^{-\lambda \alpha \tau}}{1-\mathrm{e}^{-\lambda \alpha r}}$ and finally we have

$$
\left(2 K_{1}-1\right) \leqq 2 K_{1} \delta^{\alpha} Z(2 r) \int_{0}^{2 r} k_{3}(\sigma, \delta) \mathrm{d} \sigma \frac{1}{1-\mathrm{e}^{-\lambda \alpha r}} .
$$

Now we can choose $\delta>0$ so small that neither (2.11) nor (2.15) hold, which contradicts the assumption of the existence of $t_{0}>\tau$ satisfying (2.8) and (2.9). Hence the inequality (2.7) must hold.

If $U$ is an arbitrary solution of $(0.1)$ on $\langle\tau, \tau+L)$ such that $\|U(\tau)\| \mid<\delta / 2 K_{1}$ then the function $\bar{U}(t)=\mathrm{e}^{\lambda t} U(t)$ is a solution of the equation $(2.3)$ on $\langle\tau, \tau+L)$ and $\left\||\|\bar{U}(\tau)\||<\delta \mathrm{e}^{\lambda \tau} / 2 K_{1}\right.$, i.e. in virtue of $(2.7)$ it is

$$
\|U(t)\|<2 K_{1}\|U(\tau)\| \mathrm{e}^{-\lambda(t-\tau)} .
$$

It means that the zero solution of the equation (0.1) is uniformly exponentially stable.

Proof of Lemma 2.1. Functions $\tilde{U}$ and $\tilde{V}$ satisfy the relation

$$
\begin{aligned}
\tilde{U}(t)-\tilde{V}(t) & =\int_{T}^{t} \mathrm{e}^{A(t-\sigma)}[B(\sigma)+\lambda I][\tilde{U}(\sigma)-\tilde{V}(\sigma)] \mathrm{d} \sigma+ \\
& +\int_{T}^{t} \mathrm{e}^{A(t-\sigma)} \mathrm{e}^{\lambda \sigma} N(\sigma)\left(\mathrm{e}^{-\lambda \sigma} \tilde{U}\right) \mathrm{d} \sigma .
\end{aligned}
$$


In view of (1.4) and (1.5), we get the estimate

$$
\begin{gathered}
\|\tilde{U}(t)-\tilde{V}(t)\| \leqq \int_{T}^{t} k_{2}(t-\sigma)\left(1 \times \frac{\lambda c_{2}}{c_{1}}\right)\|\tilde{U}(\sigma)-\tilde{V}(\sigma)\| \mathrm{d} \sigma+ \\
+\int_{T}^{t} \mathrm{e}^{-\lambda x \sigma} k_{3}\left(t-\sigma, \mathrm{e}^{-\lambda \sigma}\left\|\widehat{C}_{(\sigma)}(\sigma)\right\|\right)\|\tilde{U}(\sigma)\|^{1+\alpha} \mathrm{d} \sigma .
\end{gathered}
$$

Now we choose a natural number $n_{0}$ so that $2 n^{n o} /\left(2^{n_{0}}-1\right)<p(p$ is the number from the assumption (vi)) and set $2^{n_{0}} /\left(2^{n_{0}}-1\right)=p^{\prime}, 2^{n_{0}}=q^{\prime}$. Using the Hölder inequality, we have

$$
\begin{aligned}
\|\tilde{U}(t)-\tilde{V}(t)\| ! \leqq & \left(1+\frac{\lambda c_{2}}{c_{1}}\right)\left[\int_{T}^{t} k_{2}^{p^{\prime}}(t-\sigma) \mathrm{d} \sigma\right]^{1 / p^{\prime}}\left[\left.\int_{T}^{t}\|\tilde{U}(\sigma)-\tilde{V}(\sigma)\|\right|^{q^{\prime}} \mathrm{d} \sigma\right]^{1 / q^{\prime}}+ \\
& +\int_{T}^{t} \mathrm{e}^{-\lambda \alpha \delta} k_{3}\left(t-\sigma, \mathrm{e}^{+\lambda \delta}\|\tilde{U}(\sigma)\|\right)\|\tilde{U}(\sigma)\| \|^{1+\alpha} \mathrm{d} \sigma
\end{aligned}
$$

and regarding the inequality $(a+b)^{q^{\prime}} \leqq 2^{q^{\prime}-1}\left(a^{q^{\prime}}+b^{q^{\prime}}\right)$, we obtain

$$
\begin{gathered}
\|\tilde{U}(t)-\tilde{V}(t)\| \|^{q^{\prime}} \leqq 2^{q^{\prime}-1}\left(1+\frac{\lambda c_{2}}{c_{1}}\right)^{q^{\prime}}\left[\int_{T}^{t} k_{2}^{p^{\prime}}(t-\sigma) \mathrm{d} \sigma\right]^{q^{\prime} / p^{\prime}} \cdot \\
\int_{T}^{t}\|\tilde{U}(\sigma)-\tilde{V}(\sigma)\| \|^{q^{\prime}} \mathrm{d} \sigma+2^{q^{\prime}-1}\left[\int_{T}^{t} \mathrm{e}^{-\lambda \alpha \sigma} k_{3}\left(t-\sigma, \mathrm{e}^{-\lambda \sigma}\|\tilde{U}(\sigma)\|\right)\|\tilde{U}(\sigma)\| \|^{1+\alpha} \mathrm{d} \sigma\right]^{q^{\prime}} .
\end{gathered}
$$

Since $(\vartheta-T) \leqq(t-T)$ for $\vartheta \in\langle T, t\rangle$, it is

$$
\begin{gathered}
\|\tilde{U}(\vartheta)-\tilde{V}(\vartheta)\|^{q^{\prime}} \leqq 2^{q^{\prime}-1}\left(1+\frac{\lambda c_{2}}{c_{1}}\right)^{q^{\prime}}\left[\int_{T}^{t} k_{2}^{p^{\prime}}(t-\sigma) \mathrm{d} \sigma\right]^{q^{\prime} / p^{\prime}} . \\
\int_{T}^{\vartheta}\|\tilde{U}(\sigma)-\tilde{V}(\sigma)\| \|^{q^{\prime}} \mathrm{d} \sigma-2^{q^{\prime}-1}\left[\int_{T}^{t} \mathrm{e}^{-\lambda \alpha \sigma} k_{3}\left(t-\sigma, \mathrm{e}^{-\lambda \sigma}\|\tilde{U}(\sigma)\| \|\right)\|\tilde{U}(\sigma)\| \|^{1+\alpha} \mathrm{d} \sigma\right]^{q^{\prime}} .
\end{gathered}
$$

Applying the generalized Bellman inequality, we can derive that

$$
\begin{gathered}
\left.\|\tilde{U}(\vartheta)-\tilde{V}(\vartheta)\|\right|^{q^{\prime}} \leqq 2^{q^{\prime}-1}\left[\int_{T}^{\vartheta} \mathrm{e}^{-\lambda \alpha \sigma} k_{3}\left(t-\sigma, \mathrm{e}^{-\lambda \sigma}\|\tilde{U}(\sigma)\|\right)\|\tilde{U}(\sigma)\| \|^{1+\alpha} \mathrm{d} \sigma\right]^{q^{\prime}}+ \\
+\int_{T}^{\vartheta} 2^{q^{\prime}-1}\left[\left.\int_{T}^{s} \mathrm{e}^{-\lambda \alpha \sigma} k_{3}\left(t-\sigma, \mathrm{e}^{-\lambda \sigma}\|\tilde{U}(\sigma)\|\right)\|\tilde{U}(\sigma)\|\right|^{1+\alpha} \mathrm{d} \sigma\right]^{q^{\prime}} \cdot \\
.2^{q^{\prime}-1}\left(1+\frac{\lambda c_{2}}{c_{1}}\right)^{q^{\prime}}\left[\int_{T}^{t} k_{2}^{p^{\prime}}(t-\sigma) \mathrm{d} \sigma\right]^{q^{\prime} / p^{\prime}} \exp \left[2^{q^{\prime}-1}\left(1+\frac{\lambda c_{2}}{c_{1}}\right)^{q^{\prime}} .\right. \\
\left.\cdot\left(\int_{T}^{t} k_{2}^{p^{\prime}}(t-\sigma) \mathrm{d} \sigma\right)^{q^{\prime} / p^{\prime}}(s-T)\right] \mathrm{d} s
\end{gathered}
$$


and using the integration by parts on the right hand side of this inequality, we get

$$
\begin{gathered}
\|\tilde{U}(\vartheta)-\tilde{V}(\vartheta)\| \|^{q^{\prime}} \leqq 2^{q^{\prime}-1}\left[\int_{T}^{\vartheta} \mathrm{e}^{-\lambda \alpha \sigma} k_{3}\left(t-\sigma, \mathrm{e}^{-\lambda \sigma}\|\tilde{U}(\sigma)\|\right)\|\tilde{U}(\sigma)\| \|^{1+\alpha} \mathrm{d} \sigma\right]^{q^{\prime}}+ \\
+\exp \left\{2^{q^{\prime}-1}\left(1+\frac{\lambda c_{2}}{c_{1}}\right)^{q^{\prime}}\left[\int_{T}^{t} k_{2}^{p^{\prime}}(t-\sigma) \mathrm{d} \sigma\right]^{q^{\prime} / p^{\prime}}(\vartheta-T)\right\} .
\end{gathered}
$$

The last inequality was derived for all $\vartheta \in\langle T, t\rangle$. If we set $\vartheta=t$, we get (2.10) where

$$
Z(t-T)=2^{\left(q^{\prime}-1\right) / q^{\prime}} \exp \left\{2^{q^{\prime}-1}\left(1+\frac{\lambda c_{2}}{c_{1}}\right)^{q^{\prime}}\left[\int_{T}^{t} k_{2}^{p^{\prime}}(t-\sigma) \mathrm{d} \sigma\right]^{q^{\prime} / p^{\prime}}(t-T) / q^{\prime}\right\}
$$

\section{§3. THE UNIFORM STABILITY AT CONSTANTLY ACTING DISTURBANCES}

Definition 3.1. The zero solution of the equation (0.1) is uniformly stable at constantly acting disturbances if to an arbitrarily chosen $\eta>0$ there exist $\delta_{1}>0$ and $\delta_{2}>0$ so that if $\left\{H(t)\right.$ is any operator from $X_{1}$ to $\mathscr{D}\left(\|\cdot\|_{0}\right.$ ) (depending generally on $t)$ and $U$ is any solution of the equation

$$
\frac{\mathrm{d} U}{\mathrm{~d} t}=A U+B(t) U+N(t) U+H(t) U
$$

on an interval $\left\langle\tau, \tau+L\right.$ ) (where $L \in(0,+\infty\rangle$ ) such that $\|U(\tau)\|<\delta_{1}$ and $\|H(t) U(t)\|_{0}<\delta_{2}$ for all $t \in\langle\tau, \tau+L)$ which satisfy $\left.\|U(t)\|<\eta\right\}$ then the inequality $\|U(t)\|<\eta$ holds for all $t \in\langle\tau . \tau+L)$.

Remark 3.1. The number $L$ in Definition 3.1 depends on the solution $U$. The uniform stability at constantly acting disturbances is sometimes defined in such a way that the inequality $\|H(t) U(t)\|_{0}<\delta_{2}$ is required to be fulfilled for all $t \in$ $\epsilon\langle\tau, \tau+L)$ and not only for such $t \in\langle\tau, \tau+L)$ which satisfy $\|U(t)\| \mid<\eta$. However, then the stability at constantly acting disturbances is a weaker property than that defined in Definition 3.1.

Theorem 3.1. Let the zero solution of the linear equation (0.2) be uniformly exponentially stable. Then the zero solution of the equation (0.1) is uniformly stable at constantly acting disturbances.

Proof. I. First we shall prove that the zero solution of the linear equation $(0.2)$ is uniformly stable at constantly acting disturbances. Let $\eta>0$ be given. Put $\delta_{1}=\eta / 2 K_{1}\left(K_{1}\right.$ and $K_{2}$ are the numbers from the definition of the uniform exponential stability of the zero solution of $(0.2))$. Suppose that $\tau$ is an arbitrary nonnegative number and $U$ is a solution of the equation

$$
\frac{\mathrm{d} U}{\mathrm{~d} t}=A U+B(t) U+\hat{H}(t) U
$$


on $\langle\tau, \tau+L)$ such that $\|U(\tau)\|<\delta_{1} . \hat{H}(t)$ is an operator from $X_{1}$ into $\mathscr{D}\left(\|\cdot\|_{0}\right)$ If the inequality $\|U(t)\|<\delta_{1}$ does not hold for all $t \in\langle\tau, \tau+L)$ then there exists $T \in(\tau, \tau+L)$ such that $\|U(T)\|=\delta_{1}$ and $\|U(t)\|<\delta_{1}$ for all $t \in\langle\tau, T)$. Let $V$ be such a solution of $(0.2)$ on $\langle\tau,+\infty)$ that $V(T)=U(T)$. Then it may be derived in a similar way as in the case of the inequality (2.10) that

$$
\|U(t)-V(t)\| \leqq \frac{c_{2}}{c_{1}} Z(t-T) \int_{T}^{t} k_{2}(t-\sigma)\|\hat{H}(\sigma) U(\sigma)\|_{0} \mathrm{~d} \sigma,
$$

where $Z$ is the same function as in (2.10). Suppose that $\|\hat{H}(\sigma) U(\sigma)\|_{0}<\delta_{2}$ (where $\delta_{2}$ will be specified later) for all $\sigma \geqq \tau$ satisfying $\|U(\sigma)\|<\eta$. Let $t \geqq T$ be such a number that $\|U(\sigma)\|\langle\eta$ for all $\sigma \in\langle T, t\rangle$. Then by (3.3) we have

$$
\begin{aligned}
& \|U(t)\| \leqq\|V(t)\|+\frac{c_{2}}{c_{1}} Z(t-T) \delta_{2} \int_{T}^{t} k_{2}(t-\sigma) \mathrm{d} \sigma \leqq \\
& \leqq K_{1}\|V(T)\| \mathrm{e}^{-K_{2}(t-T)}+\frac{c_{2}}{c_{1}} Z(t-T) \delta_{2} \int_{0}^{t-T} k_{2}(\sigma) \mathrm{d} \sigma .
\end{aligned}
$$

It can be shown that there exist $t_{0}>0$ and $C>0$ so that

$$
\begin{gathered}
K_{1} \mathrm{e}^{-K_{2} t_{0}}+\frac{c_{2}}{c_{1}} Z\left(t_{0}\right) C \int_{0}^{t_{0}} k_{2}(\sigma) \mathrm{d} \sigma<1, \\
K_{1} \mathrm{e}^{-K_{2} s}+\frac{c_{2}}{c_{1}} Z(s) C \int_{0}^{s} k_{2}(\sigma) \mathrm{d} \sigma<2 K_{1} \text { for } s \in\left\langle 0, t_{0}\right\rangle .
\end{gathered}
$$

Put

$$
\delta_{2}=C \delta_{1}\left(\equiv C \eta / 2 K_{1}\right) .
$$

In virtue of (3.4) -(3.6) we have $\|U(\sigma)\|\left\langle\eta\right.$ for all $\sigma \in\left\langle T, T+t_{0}\right\rangle \cap\langle\tau, \tau+L)$. But if $T+t_{0}<\tau+L$ then by (3.5) it is $\left\|U\left(T+t_{0}\right)\right\|<\delta_{1}$ as well and hence we can derive in the same way that the inequality $\|U(\sigma)\|<\eta$ holds also for all $\sigma \in$ $\in\left\langle T+t_{0}, T+2 t_{0}\right\rangle \cap\left\langle T+t_{0}, \tau+L\right)$ and consequently for all $\sigma \in\langle T, \tau+L)$.

II. We shall now prove the uniform stability at constantly acting disturbances of the zero solution of the equation (0.1). Put $\hat{H}(t) U=H(t) U+N(t) U$. Let $\eta^{\prime}$ be an arbitrary positive number. There exist $\eta \in\left(0, \eta^{\prime}\right)$ and $\delta_{2}^{\prime}>0$ so that

$$
k_{4}(\eta) \eta^{1+\alpha}+\delta_{2}^{\prime}<C \eta / 2 K_{1}
$$

(where $C$ is the number from (3.5)). Put $\delta_{1}^{\prime}=\eta / 2 K_{1}$.

If $U$ is a solution of the equation (3.1) on $\langle\tau, \tau+L)$ and $\|U(\tau)\|<\delta_{1}^{\prime}, \| H(t)$ $U(t) \|_{0}<\delta_{1}^{\prime}$ for all $t \geqq \tau$ satisfying $\|U(t)\|<\eta^{\prime}$ then by the inequality $\eta \leqq \eta^{\prime}$ it is $\|H(t) U(t)\|_{0}<\delta_{2}^{\prime}$ for all $t \in\langle\tau, \tau+L)$ satisfying $\|U(t)\|<\eta$. But this implies that $\|\hat{H}(t) U(t)\|_{0} \leqq k_{4}(\eta) \eta^{1+\alpha}+\delta_{2}^{\prime}$ for $t \in\langle\tau, \tau+L)$ satisfying $\|U(t)\|<\eta$. By (3.8) 
we have $\|\hat{H}(t) U(t)\|_{0}<C \eta / 2 K_{1}$ and hence, considering (3.5), also $\|\hat{H}(t) U(t)\|_{0}<$ $<\delta_{2}$ for those $t$, too. Using the results of part I of this proof we obtain the estimate $\|U(t)\|<\eta$ for all $t \in\langle\tau, \tau+L)$, i.e. also $\|U(t)\|<\eta^{\prime}$ for all $t \in\langle\tau, \tau+L)$.

\section{§ 4. APPLICATIONS}

\subsection{THE NAVIER-STOKES EQUATIONS}

The flow of a viscous incompressible fluid in a domain $\Omega$ is described by the Navier-Stokes equations

$$
\frac{\partial \boldsymbol{U}}{\partial t}+(\boldsymbol{U}, \nabla) \boldsymbol{U}=\boldsymbol{F}-\frac{1}{\varrho} \operatorname{grad} p+v \Delta \boldsymbol{U}
$$

and the equation of continuity

$$
\operatorname{div} \boldsymbol{U}=0,
$$

where $\boldsymbol{U}$ is the velocity of the flow, $p$ is the pressure, $\boldsymbol{F}$ is the body force, $\varrho$ is the density of the fluid and $v$ is the kinematic coefficient of viscosity.

Suppose that $\Omega$ is a bounded domain in $E_{3}$ with a lipschitzian boundary $\partial \Omega$. We shall denote by $\mathscr{E}(\Omega)$ the space of infinitely differentiable vector-functions $\boldsymbol{U}$ defined in $\Omega$ and such that $\operatorname{div} \boldsymbol{U}=0$ in $\Omega$, and by $\mathscr{D}(\Omega)$ the space of all functions from $\mathscr{E}(\Omega)$ which have a compact support in $\Omega$.J $(\Omega)$ or $J_{2}(\Omega)$ will be the closure of $\mathscr{E}(\Omega)$ in $L_{2}(\Omega)$ or in $W_{2}^{2}(\Omega)$, respectively, while ${ }^{0} J(\Omega)$ or ${ }^{0} J_{1}(\Omega)$ will be the closure of $\mathscr{D}(\Omega)$ in $L_{2}(\Omega)$ or in $W_{2}^{1}(\Omega)$, respectively. Let us denote $A=\nu P_{\sigma} * \Delta$, where $P_{\sigma}$ is the orthogonal projection of $L_{2}(\Omega)$ onto ${ }^{0} J(\Omega)$ and $\Delta$ is the Laplace operator. The operator $A$ will be considered on $\mathscr{D}(A)={ }^{0} \boldsymbol{J}_{1}(\Omega) \cap \boldsymbol{W}_{2}^{2}(\Omega)$. Put $X={ }^{0} J(\Omega)$ and following $\S 1$, let $X_{1}$ be the Banach space $\mathscr{D}(A)$ with the norm $\|\cdot\|_{1}=\|\cdot\|_{0}+\|A \cdot\|_{0}$ (where $\|\cdot\|_{0}$ is the norm in $\left.{ }^{0} J(\Omega)\right)$. The norm in ${ }^{0} J_{1}(\Omega)$ will be denoted $\|\cdot\|_{J_{J_{1}}}$. It is shown in [6] and [7] that the operator $A$ is the generator of a semigroup of operators $\mathrm{e}^{A t}$ of the class $C_{0}$ in $X$ and, in addition to other properties, $\mathrm{e}^{A t} x \in X_{1}$ for $x \in{ }^{0} J_{1}(\Omega)$ and there exists a nondecreasing function $\varkappa$ on $(0,+\infty)$ so that

$$
\left\|\mathrm{e}^{A t} \mathbf{x}\right\|_{1} \leqq \varkappa(l)\|\mathbf{x}\|_{0_{J_{1}}} \mid t^{3 / 4}
$$

for all $l>0, t \in(0, l\rangle$ and $\mathbf{x} \in \boldsymbol{J}^{0} \boldsymbol{J}_{1}(\Omega)$

Suppose that $\boldsymbol{U}_{0} \in C^{1}(\langle 0,+\infty) ; J(\Omega)) \cap C^{0}\left(\langle 0,+\infty) ; J_{2}(\Omega)\right)$ is a solution of the system of equations (4.1.1) and (4.1.2), satisfying the boundary condition

$$
\left.\boldsymbol{U}_{0}(t)\right|_{\partial \Omega}=\boldsymbol{\Psi}(t) \quad(\text { for } t \geqq 0) .
$$

The stability of the solution $\boldsymbol{U}_{0}$ depends on the behvaiour of differences $\boldsymbol{U}(t)-\boldsymbol{U}_{0}(t)$ 
for every solution $\boldsymbol{U} \in \mathscr{C}^{1}(\langle\tau, \tau+L) ; J(\Omega)) \cap \mathscr{C}^{0}\left(\langle\tau, \tau+L) ; J_{2}(\Omega)\right.$ ) (where $\tau \geqq 0$, $L \in(0,+\infty\rangle)$ of the system (4.1.1) and (4.1.2), satisfying (4.1.5). Substituting $V(t)=$ $=\mathbf{U}(t)-\mathbf{U}_{0}(t)$ we see that $\boldsymbol{V}$ solves the system of equations

$$
\frac{\partial \boldsymbol{V}}{\partial t}+\left(\boldsymbol{U}_{0}, \nabla\right) \boldsymbol{V}+(\boldsymbol{V}, \nabla) \boldsymbol{U}_{0}+(\boldsymbol{V}, \nabla) \boldsymbol{V}=-\frac{1}{\varrho} \operatorname{grad} p^{\prime}+v \Delta \boldsymbol{V}, \quad \operatorname{div} \boldsymbol{V}=0
$$

and satisfies the boundary condition

$$
\left.\mathbf{V}(t)\right|_{\partial \Omega}=\mathbf{0} \quad(\text { for } t \in\langle\tau, \tau+L)) .
$$

It follows from (4.1.7) that $\boldsymbol{V}(t) \in X_{1}$ and $\partial \mathbf{V}(t) / \partial t \in X$ for $t \in\langle\tau, \tau+L$ ), i.e. $\boldsymbol{V} \in$ $\in \mathscr{C}^{1}(\langle\tau, \tau+L) ; X) \cap \mathscr{C}^{0}\left(\langle\tau, \tau+L) ; X_{1}\right)$. Also it is seen that the stability of the solution $\boldsymbol{U}_{0}$ of (4.1.1), (4.1.2) and (4.1.5) is equivalent to the stability of the zero solution of (4.1.6), (4.1.7).

Applying the projection $P_{\sigma}$ to the system (4.1.6) and denoting

$$
B(t) \boldsymbol{V}=-P_{\sigma}\left[\left(\mathbf{U}_{0}(t), \nabla\right) \boldsymbol{V}+(\boldsymbol{V}, \nabla) \boldsymbol{U}_{0}(t)\right], \quad N \boldsymbol{V}=-P_{\sigma}[(\boldsymbol{V}, \nabla) \boldsymbol{V}],
$$

we get the equation $(0.1)$. The term $P_{\sigma} \operatorname{grad} p^{\prime}$ is equal to zero, because grad $p^{\prime}$ is orthogonal to each element of ${ }^{0} J(\Omega)$ in $L_{2}(\Omega)$ (see e.g. [11]). It may be easily verified that a function $\boldsymbol{V} \in \mathscr{C}^{1}(\langle\tau, \tau+L) ; X) \cap \mathscr{C}^{0}\left(\langle\tau, \tau+L) ; X_{1}\right)$ satisfies (4.1.6) and (4.1.7) if and only if it is a solution of the equation (0.1) on the interval $\langle\tau, \tau+L)$.

The operators $B(t)$ and $N$ are defined on ${ }^{0} J_{1}(\Omega)$. Using the embedding theorem (see e.g. [11], p. 33), the Hölder inequality and the inequality $\|\mathbf{x}\|_{\mathrm{O}_{2}(\Omega)} \leqq$ const. $\|\mathbf{x}\|_{1}$ which is for example a consequence of Theorem 3 in [11], p. 102, we can derive that there exists $c_{4}>1$ such that

$$
\|B(t) \mathbf{x}\|_{\mathrm{o}_{\boldsymbol{J}_{1}}} \leqq c_{4}\|\mathbf{x}\|_{1}, \quad\|N \mathbf{x}\|_{\mathrm{O}_{J_{1}}} \leqq c_{4}\|\mathbf{x}\|_{1}^{2}
$$

for all $t \geqq 0$ and $\mathbf{x} \in X_{1}$. Thus, it follows from (4.1.3) and (4.1.8) that if we put $k_{1}(t)=c_{4} x(t) / t^{3 / 4}$ and $\alpha=1$ then the conditions (v) and (vii) from $\S 1$ are fulfilled. Further, put $\|\cdot \mid\|=\|\cdot\|_{1},\|\cdot\|_{0}=\|\cdot\|_{\mathrm{o}_{1}}, \mathscr{D}\left(\|\cdot\|_{0}\right)={ }^{0} J_{1}(\Omega), k_{2}(t)=c_{4} x(t) / t^{3 / 4}$ and let $p$ be an arbitrary number from the interval $(1,4 / 3)$. Then also the inequality (1.3) and the condition (vi) from $\S 1$ are fulfilled.

Hence we can use Theorem 2.1 and Theorem 3.1 and conclude that if the zero solution of the linear equation (0.2) is uniformly exponentially stable then the zero solution of the equation $(0.1)$ has the same property and also it is uniformly stable at constantly acting disturbances. On the other hand, the uniform exponential stability of the zero solution of the equation (0.1) is equivalent to the same property of the solution $\boldsymbol{U}_{0}$ of (4.1.1), (4.1.2), (4.1.5) and it may be easily proved that the uniform stability at constantly acting disturbances of the zero solution of the equation (0.1) implies that the solution $\boldsymbol{U}_{0}$ of $(4.1 .1),(4.1 .2),(4.1 .5)$ has this property as well. 
Besides, taking into account the relation between the equation $(0.2)$ and the system

$$
\begin{gathered}
\frac{\partial \boldsymbol{V}}{\partial t}+(\boldsymbol{V}, \nabla) \boldsymbol{U}_{0}+\left(\boldsymbol{U}_{0}, \nabla\right) \boldsymbol{V}=-\frac{1}{\varrho} \operatorname{grad} p^{\prime \prime}+v \Delta \boldsymbol{V}, \quad \operatorname{div} \boldsymbol{V}=0 \\
\left.\boldsymbol{V}(t)\right|_{i \Omega}=\mathbf{0}
\end{gathered}
$$

we can write:

Theorem 4.1.1. Let the zero solution of the system (4.1.9), (4.1.10) be uniformly exponentially stable. Then the solution $\mathbf{U}_{0}$ of the system of equations (4.1.1) and (4.1.2) with the boundary condition (4.1.5) is uniformly exponentially stable and uniformly stable at constantly acting disturbances.

Remark 4.1.1. If the assumptions of Theorem 4.1.1 are modified in such a way that we require the zero solution of (4.1.9) to be uniformly exponentially stable regardless of the boundary condition (4.1.10) (i.e. not only solutions of (4.1.9) satisfying the boundary condition (4.1.10), but all solutions of (4.1.9) are exponentially decreasing to zero as $t \rightarrow+\infty$ ) then it may be proved that the solution $\boldsymbol{U}_{0}$ of the system (4.1.1) and (4.1.2) is uniformly exponentially stable and uniformly stable at constantly acting disturbances (i.e. roughly speaking we have information about the behaviour of differences $\boldsymbol{U}(t)-\boldsymbol{U}_{0}(t)$ as $t \rightarrow+\infty$ in the case of all solutions $\boldsymbol{U}$ and not only in the case of those solutions $\boldsymbol{U}$ which satisfy the boundary condition (4.1.5)). The proof of this result is quite analogous to the proofs of Theorems 2.1 and 3.1. The only differences are: a) We work with the systems of equations (4.1.1), (4.1.2) and (4.1.9) instead of the equations (0.1) and (0.2). b) Given a solution $\boldsymbol{U}$ of the system (4.1.1), (4.1.2) on $\langle\tau,+\infty)$, we cannot use Lemma 1.3 in order to establish the existence of a solution $\boldsymbol{V}$ of (4.1.9) on $\langle\tau,+\infty)$ such that $\boldsymbol{V}(\tau)=\mathbf{U}(\tau)$, $\left.\mathbf{V}(t)\right|_{\partial \Omega}=\left.\mathbf{U}(t)\right|_{\partial \Omega}($ for $t \geqq \tau)$. Nonetheless, using Lemma 3 in [7], p. 47, we can prove that to a given solution $\boldsymbol{U}$ of $(4.1 .1),(4.1 .2)$ on $\langle\tau,+\infty)$ there exists a solution $\mathbf{W} \in \mathscr{C}^{1}(\langle\tau,+\infty) ; X) \cap \mathscr{C}^{0}\left(\langle\tau,+\infty) ; X_{1}\right)$ of the system

$$
\begin{gathered}
\frac{\partial \mathbf{W}}{\partial t}+(\mathbf{W}, \nabla) \mathbf{U}_{0}+\left(\mathbf{U}_{0}, \nabla\right) \mathbf{W}=-\frac{1}{\varrho} \operatorname{grad} p+v \Delta \mathbf{W}+(\mathbf{U}, \nabla) \mathbf{U}, \\
\operatorname{div} \mathbf{W}=0
\end{gathered}
$$

such that $\mathbf{W}(\tau)=\mathbf{0},\left.\mathbf{W}\right|_{\partial \Omega}=\mathbf{0}$ and then $\boldsymbol{V}=\boldsymbol{U}+\mathbf{W}$ is the solution of (4.1.9) that we are looking for. c) The difference, $\widetilde{\boldsymbol{U}}-\tilde{\boldsymbol{V}}$ (see Lemma 2.1) is an element of $\mathscr{C}^{1}(\langle T,+\infty) ; X) \cap \mathscr{C}^{0}\left(\langle T,+\infty) ; X_{1}\right)$ and satisfies the equation

$$
\frac{\mathrm{d}(\tilde{\mathbf{U}}-\tilde{\mathbf{V}})}{\mathrm{d} t}=A(\tilde{\mathbf{U}}-\tilde{\mathbf{V}})+B(t)(\tilde{\mathbf{U}}-\tilde{\mathbf{V}})+\lambda(\tilde{\mathbf{U}}-\tilde{\mathbf{V}})+N\left(\mathrm{e}^{-\lambda t} \tilde{\mathbf{U}}\right)
$$

hence it satisfies the relation (2.16) and also the inequality (2.10). 
Remark 4.1.2. The same results as in Theorem 4.1.1 and Remark 4.1.1 could be proved also if we put $X=C^{(h)}(\bar{\Omega}),\|\| \cdot\left\|\left.|=| \cdot\right|_{2+(h)},\right\||\cdot| \|_{0}=|\cdot|_{1+(h)}$, where $h \in(0,1)$ and $C^{(h)}(\bar{\Omega})$ is the Banach space of continuous vector functions $f=\left[f_{1}, f_{2}, f_{3}\right]$ defined on $\bar{\Omega}$, with the norm $|\cdot|_{0+(h)}$. (The norm $|\cdot|_{l+(h)}(l=0,1,2)$ is defined as follows:

$$
\begin{gathered}
|\boldsymbol{f}|_{l+(h)}=\sum_{k=0}^{l} \sum_{|m|=k} \max _{x \in \Omega}\left[\sum_{i=1}^{3}\left(D^{m} f_{i}(x)^{2}\right]^{1 / 2}+\right. \\
\left.+\sum_{|m|=l} \max _{x_{1}, x_{2} \in \bar{\Omega}} \frac{\left[\sum_{i=1}^{3}\left(D^{m} f_{i}\left(x_{1}\right)-D^{m} f_{i}\left(x_{2}\right)\right)^{2}\right]^{1 / 2}}{\left|x_{1}-x_{2}\right|^{h}} .\right)
\end{gathered}
$$

\subsection{THE WAVE EQUATION}

Let $u_{0}$ be a solution of the problem given by the wave equation

$$
u_{t t}-u_{x x}=F\left(t, x, u, u_{t}, u_{x}\right) \quad(t \in\langle 0,+\infty), x \in\langle 0, \pi\rangle)
$$

and by one of boundary conditions

$$
\begin{gathered}
u(t, 0)=u(t, \pi)=0 \quad(\text { for } t \geqq 0), \\
u_{x}(t, 0)+\alpha_{0} u(t, 0)=u(t, \pi)=0 \quad(\text { for } t \geqq 0)
\end{gathered}
$$

and

$$
u_{x}(t, 0)+\alpha_{0} u(t, 0)=u_{x}(t, \pi)+\alpha_{\pi} u(t, \pi)=0 \quad(\text { for } t \geqq 0) .
$$

In the following the symbol $x$ means the space variable and is not used for elements of some Banach spaces as in the previous sections.

We shall sometimes denote $u_{1}=u, u_{2}=u_{t}, u_{3}=u_{x}$. The function $F$ is supposed to satisfy the following conditions:

$\left(\mathscr{A}_{1}\right) F$ together with its partial derivatives

$$
\frac{\partial F}{\partial x}, \frac{\partial F}{\partial u_{i}}, \frac{\partial^{2} F}{\partial u_{i} \partial x}, \frac{\partial^{2} F}{\partial u_{i} \partial u_{j}}, \frac{\partial^{3} F}{\partial u_{i} \partial u_{j} \partial x} \quad(i, j=1,2,3)
$$

is continuous and bounded for $t \geqq 0, x \in\langle 0, \pi\rangle,\left[u_{1}, u_{2}, u_{3}\right] \in\langle-R, R\rangle \times$ $\times\langle-R, R\rangle \times\langle-R, R\rangle$ ( $R$ being an arbitrary positive number),

$\left(\mathscr{A}_{2}\right) F\left(t, 0,0,0, u_{x}\right)=F\left(t, \pi, 0,0, u_{x}\right)=0$ or $F\left(t, \pi, 0,0, u_{x}\right)=0$ for $t \geqq 0, u_{x} \in E_{1}$ in the case of the boundary conditions (4.2.2) or (4.2.3), respectively.

Further, we shall study the equation (4.2.1) with boundary conditions (4.2.2) and

$$
\begin{array}{ll}
u_{x}(t, 0)=u(t, \pi)=0 & (\text { for } t \geqq 0) \\
u_{x}(t, 0)=u_{x}(t, \pi)=0 & (\text { for } t \geqq 0)
\end{array}
$$


instead of (4.2.3) or (4.2.4), respectively, without loss of generality, because it may be easily proved that if $\chi(x)$ is a sufficiently smooth function defined on $\langle 0, \pi\rangle$ and satisfying the condition

$$
\chi^{\prime}(0)=\alpha_{0}
$$

or

$$
\chi^{\prime}(0)=\alpha_{0}, \quad \chi^{\prime}(\pi)=\alpha_{\pi}
$$

then a function $u$ satisfies the equation (4.2.1) and the boundary conditions (4.2.3) or (4.2.4) if and only if the function $v=u$. $\mathrm{e}^{\chi}$ satisfies the equation

$$
v_{t t}-v_{x x}=\left(\chi^{\prime \prime}-\chi^{\prime 2}\right) v+2 \chi^{\prime} v_{x}+\mathrm{e}^{\chi} F\left(t, x, \mathrm{e}^{-\chi} v, \mathrm{e}^{-\chi} v_{t},-\chi^{\prime} \mathrm{e}^{-\chi} v+\mathrm{e}^{-\chi} v_{x}\right)
$$

and the boundary conditions (4.2.5) or (4.2.6), respectively. However, the equation (4.2.9) may be considered as an equation of the type (4.2.1).

Provided $u_{0} \in C^{2}\left(\langle 0,+\infty) ; C^{0}(\langle 0, \pi\rangle)\right) \cap C^{1}\left(\langle 0,+\infty) ; C^{1}(\langle 0, \pi\rangle)\right) \cap C^{0}(\langle 0, \infty) ;$ $\left.C^{2}(\langle 0, \pi\rangle)\right)$, the problem of the uniform exponential stability (or the stability at constantly acting disturbances) of the solution $u_{0}$ can be easily transformed to the same problem concerning the zero solution of the equation

$$
\begin{gathered}
u_{t t}-u_{x x}=a(t, x) u+b(t, x) u_{t}+c(t, x) u_{x}+ \\
+\sum_{i, j=1}^{3} d_{i j}\left(t, x, u, u_{t}, u_{x}\right) \cdot u_{i} \cdot u_{j},
\end{gathered}
$$

where

$$
\begin{gathered}
a(t, x)=\frac{\partial F}{\partial u}\left(t, x, u_{0}(t, x), u_{0 t}(t, x), u_{0 x}(t, x)\right), \\
b(t, x)=\frac{\partial F}{\partial u_{t}}\left(t, x, u_{0}(t, x), u_{0 t}(t, x), u_{0 x}(t, x)\right), \\
c(t, x)=\frac{\partial F}{\partial u_{x}}\left(t, x, u_{0}(t, x), u_{0 t}(t, x), u_{0 x}(t, x)\right), \\
d_{i j}\left(t, x, u, u_{t}, u_{x}\right)=\int_{0}^{1} \int_{0}^{1} \frac{\partial^{2} F}{\partial u_{i} \partial u_{j}}\left(t, x, u_{0}(t, x)+\alpha \beta u,\right. \\
\left.u_{0 t}(t, x)+\alpha \beta u_{t}, u_{0 x}(t, x)+\alpha \beta u_{x}\right) \beta \mathrm{d} \alpha \mathrm{d} \beta
\end{gathered}
$$

(for $i, j=1,2,3$ ). It is a consequence of $\left(\mathscr{A}_{1}\right)$ that the functions $a, b, c$ and $d_{i j}$ are continuous in all variables, continuously differentiable with respect to $x$ and bounded for $t \in\langle 0,+\infty), x \in\langle 0, \pi\rangle,\left[u, u_{t}, u_{x}\right] \in\langle-R, R\rangle \times\langle-R, R\rangle \times\langle-R, R\rangle$. The equation (4.2.10) will be of course examined with the same boundary conditions as the equation (4.2.1). It follows from the properties of the function $F$ that $c(t, 0)=$ $=c(t, \pi)=d_{33}\left(t, 0,0,0, u_{x}\right)=d_{33}\left(t, \pi, 0,0, u_{x}\right)=0$ or $c(t, \pi)=d_{33}\left(t, \pi, 0,0, u_{x}\right)=$ $=0$ if we deal with the boundary conditions (4.2.2) or (4.2.5), respectively. 
Using the notation $u_{1}=u, u_{2}=u_{t}, u_{3}=u_{x}$, we can rewirte the equation (4.2.10) in the form of the following system of equations:

$$
\begin{gathered}
u_{1 t}=u_{2} \\
u_{2 t}=u_{1 x x}+a u_{1}+b u_{2}+c u_{1 x}+\sum_{i, j=1}^{3} d_{i j} \cdot u_{i} \cdot u_{j} .
\end{gathered}
$$

Let $X$ be the space of all elements $f=\left[f_{1}, f_{2}\right] \in C^{1}(\langle 0, \pi\rangle) \times C^{0}(\langle 0, \pi\rangle)$ such that $f_{1}(0)=f_{1}(\pi)=f_{2}(0)=f_{2}(\pi)=0$ or $f_{1}^{\prime}(0)=f_{1}(\pi)=f_{2}(\pi)=0$ or $f_{1}^{\prime}(0)=$ $=f_{1}^{\prime}(\pi)=0$ if the boundary conditions (4.2.2) or (4.2.5) or (4.2.6), respectively, are considered. $X$ is a Banach space with the norm

$$
\|f\|_{0}=\max _{x \in\langle 0, \pi\rangle}\left|f_{1}(x)\right|+\max _{x \in\langle 0, \pi\rangle}\left|f_{1}^{\prime}(x)\right|+\max _{x \in\langle 0, \pi\rangle}\left|f_{2}(x)\right| .
$$

Put

$$
\mathscr{D}(A)=X \cap\left\{\left[f_{1}, f_{2}\right] \mid f_{1} \in C^{2}(\langle 0, \pi\rangle), f_{2} \in C^{1}(\langle 0, \pi\rangle), f_{1}^{\prime \prime}(0)=f_{1}^{\prime \prime}(\pi)=0\right\}
$$

or

or

$$
\mathscr{D}(A)=X \cap\left\{\left[f_{1}, f_{2}\right] \mid f_{1} \in C^{2}(\langle 0, \pi\rangle), f_{2} \in C^{1}(\langle 0, \pi\rangle), f_{2}^{\prime}(0)=f_{1}^{\prime \prime}(\pi)=0\right\}^{\prime \prime \prime}
$$

$$
\mathscr{D}(A)=X \cap\left\{\left[f_{1}, f_{2}\right] \mid f_{1} \in C^{2}(\langle 0, \pi\rangle), f_{2} \in C^{1}(\langle 0, \pi\rangle), f_{2}^{\prime}(0)=f_{2}^{\prime}(\pi)=0\right\}
$$

in the case of the boundary conditions (4.2.2) or (4.2.5) or (4.2.6), respectively, and

$$
A f=\left[f_{2}, f_{1}^{\prime \prime}\right](\text { for } f \in \mathscr{D}(A)) .
$$

It may be proved that the operator $A$ is the generator of a semigroup of operators $\mathrm{e}^{A t}$ of the class $C_{0}$ in $X$ such that

$$
\begin{gathered}
\left(\mathrm{e}^{A t} f\right)(x)=\frac{1}{2}\left[f_{1}(x+t)+f_{1}(x-t)+\int_{x-t}^{x+t} f_{2}(\sigma) \mathrm{d} \sigma\right. \\
\left.f_{1}^{\prime}(x+t)-f_{1}^{\prime}(x-t)+f_{2}(x+t)+f_{2}(x-t)\right]
\end{gathered}
$$

for $f \in X$. The symbols $f_{1}$ and $f_{2}$ in (4.2.13) mean the functions $f_{1}$ and $f_{2}$ with an extended domain of definition so that

- $f_{1}$ and $f_{2}$ are odd, $2 \pi$-periodic functions on $E_{1}$ in the case of the boundary conditions (4.2:2),

$-f_{1}$ and $f_{2}$ are even, $4 \pi$-periodic functions such that $f_{1}(x)=-f_{1}(2 \pi-x)$ for all $x \in E_{1}$ in the case of the boundary conditions (4.2.5),

- $f_{1}$ and $f_{2}$ are even, $2 \pi$-periodic functions on $E_{1}$ in the case of the boundary conditions (4.2.6). 
Denote by $X_{1}$ the space $\mathscr{D}(A)$ with the norm $\|\cdot\|_{1}=\|\cdot\|_{0}+\|A \cdot\|_{0}$, as in Section 1 . Put $\mathscr{D}\left(\|\cdot\|_{0}\right)=X,\|\cdot\|_{0}=\|\cdot\|_{0}$ and $\|\cdot\|=\|\cdot\|_{1}$. Further, put

$$
\begin{aligned}
& B(t) f(x)=\left[0, a(t, x) f_{1}(x)+b(t, x) f_{2}(x)+c(t, x) f_{1}^{\prime}(x)\right], \\
& N(t) f(x)=\left[0, \sum_{i, j=1}^{3} d_{i j}\left(t, x, f_{1}, f_{2}, f_{1}^{\prime}\right) . f_{i}(x) . f_{j}(x)\right]
\end{aligned}
$$

(we denote $f_{3}(x)=f_{1}^{\prime}(x)$ ). Now, using the notation $U=\left[u_{1}, u_{2}\right]=\left[u, u_{t}\right]$, we can write the system (4.2.11) in the form (0.1). The operators $B(t)$ and $N(t)$ map $X$ into itself and it may be verified that $\mathrm{e}^{A t} B(s) f$ and $\mathrm{e}^{A t} N(s) f$ belong to $X_{1}$ if $f \in X_{1}$. Further, if $f=\left[f_{1}, f_{2}\right] \in X_{1}$ and $t \geqq 0, s \geqq 0$, it is

$$
\begin{aligned}
& \left\|\mathrm{e}^{A t} B(s) f\right\|_{1}=\left\|A \mathrm{e}^{A t} B(s) f\right\|_{0}+\mathrm{e}^{A t} B(s) f \|_{0}= \\
& =\frac{1}{2} \| A\left[\int_{x-t}^{x+t}\left(a(s, \sigma) f_{1}(\sigma)+b(s, \sigma) f_{2}(\sigma)+c(s, \sigma) f_{1}^{\prime}(\sigma)\right) \mathrm{d} \sigma,\right. \\
& a(s, x+t) f_{1}(x+t)+b(s, x+t) f_{2}(x+t)+c(s, x+t) f_{1}^{\prime}(x+t)+ \\
& \left.+a(s, x-t) f_{1}(x-t)+b(s, x-t) f_{2}(x-t)+c(s, x-t) f_{1}^{\prime}(x-t)\right]\|\|_{0}+ \\
& +\frac{1}{2} \|\left[\int_{x-t}^{x+t}\left(a(s, \sigma) f_{1}(\sigma)+b(s, \sigma) f_{2}(\sigma)+c(s, \sigma) f_{1}^{\prime}(\sigma)\right) \mathrm{d} \sigma,\right. \\
& a(s, x+t) f_{1}(x+t)+b(s, x+t) f_{2}(x+t)+c(s, x+t) f_{1}^{\prime}(x+t)+ \\
& \left.+a(s, x-t) f_{1}(x-t)+b(s, x-t) f_{2}(x-t)+c(s, x-t) f_{1}^{\prime}(x-t)\right] \|_{0}= \\
& =\frac{1}{2} \|\left[a(s, x+t) f_{1}(x+t)+b(s, x+t) f_{2}(x+t)+c(s, x+t) f_{1}^{\prime}(x+t)+\right. \\
& +a(s, x-t) f_{1}(x-t)+b(s, x-t) f_{2}(x-t)+c(s, x-t) f_{1}^{\prime}(x-t) \text {, } \\
& a_{x}(s, x+t) f_{1}(x+t)+a(s, x+t) f_{1}^{\prime}(x+t)+b_{x}(s, x+t) f_{2}(x+t)+ \\
& +b(s, x+t) f_{2}^{\prime}(x+t)+c_{x}(s, x+t) f_{1}^{\prime}(x+t)+c(s, x+t) f_{1}^{\prime \prime}(x+t)- \\
& -a_{x}(s, x-t) f_{1}(x-t)-a(s, x-t) f_{1}^{\prime}(x-t)-b_{x}(s, x-t) f_{2}(x-t)- \\
& \left.-b(s, x-t) f_{2}^{\prime}(x-t)-c_{x}(s, x-t) f_{1}^{\prime}(x-t)-c(s, x-t) f_{1}^{\prime \prime}(x-t)\right] \|_{0}+ \\
& +\frac{1}{2} \|\left[\int_{x-t}^{x+t}\left(a(s, \sigma) f_{1}(\sigma)+b(s, \sigma) f_{2}(\sigma)+c(s, \sigma) f_{1}^{\prime}(\sigma)\right) \mathrm{d} \sigma,\right. \\
& a(s, x+t) f_{1}(x+t)+b(s, x+t) f_{2}(x+t)+c(s, x+t) f_{1}^{\prime}(x+t)+ \\
& \left.+a(s, x-t) f_{1}(x-t)+b(s, x-t) f_{2}(x-t)+c(s, x-t) f_{1}^{\prime}(x-t)\right] \|_{0}= \\
& =\frac{1}{2} \max _{x \in\langle 0, \pi\rangle} \mid a(s, x+t) f_{1}(x+t)+b(s, x+t) f_{2}(x+t)+c(s, x+t) f_{1}^{\prime}(x+t)+
\end{aligned}
$$




$$
\begin{aligned}
& \quad+a(s, x-t) f_{1}(x-t)+b(s, x-t) f_{2}(x-t)+c(s, x-t) f_{1}^{\prime}(x-t) \mid+ \\
& +\frac{1}{2} \max _{x \in\langle 0, \pi\rangle} \mid a_{x}(s, x+t) f_{1}(x+t)+a(s, x+t) f_{1}^{\prime}(x+t)+b_{x}(s, x+t) f_{2}(x+t)+ \\
& \quad+b(s, x+t) f_{1}^{\prime}(x+t)+c_{x}(s, x+t) f_{1}^{\prime}(x+t)+c(s, x+t) f_{1}^{\prime \prime}(x+t)+ \\
& +a_{x}(s, x-t) f_{1}(x-t)+a(s, x-t) f_{1}^{\prime}(x-t)+b_{x}(s, x-t) f_{2}(x-t)+ \\
& \quad+b(s, x-t) f_{2}^{\prime}(x-t)+c_{x}(s, x-t) f_{1}^{\prime}(x-t)+c(s, x-t) f_{1}^{\prime \prime}(x-t) \mid+ \\
& +\frac{1}{2} \max _{x \in\langle 0, \pi\rangle} \mid a_{x}(s, x+t) f_{1}(x+t)+a(s, x+t) f_{1}^{\prime}(x+t)+b_{x}(s, x+t) f_{2}(x+t)+ \\
& \quad+b(s, x+t) f_{2}^{\prime}(x+t)+c_{x}(s, x+t) f_{1}^{\prime}(x+t)+c(s, x+t) f_{1}^{\prime \prime}(x+t)- \\
& \quad-a_{x}(s, x-t) f_{1}(x-t)-a(s, x-t) f_{1}^{\prime}(x-t)-b_{x}(s, x-t) f_{2}(x-t)- \\
& \quad-b(s, x-t) f_{2}^{\prime}(x-t)-c_{x}(s, x-t) f_{1}^{\prime}(x-t)-c(s, x-t) f_{1}^{\prime \prime}(x-t) \mid+ \\
& \quad+\frac{1}{2} \max _{x \in\langle 0, \pi\rangle}\left|\int_{x-t}^{x+t}\left(a(s, \sigma) f_{1}(\sigma)+b(s, \sigma) f_{2}(\sigma)+c(s, \sigma) f_{1}^{\prime}(\sigma)\right) d \sigma\right|+ \\
& +\frac{1}{2} \max _{x \in\langle 0, \pi\rangle} \mid a(s, x+t) f_{1}(x+t)+b(s, x+t) f_{2}(x+t)+c(s, x+t) f_{1}^{\prime}(x+t)- \\
& \quad-a(s, x-t) f_{1}(x-t)-b(s, x-t) f_{2}(x-t)-c(s, x-t) f_{1}^{\prime}(x-t) \mid+ \\
& +\frac{1}{2} \max _{x \in\langle 0, \pi\rangle} \mid a(s, x+t) f_{1}(x+t)+b(s, x+t) f_{2}(x+t)+c(s, x+t) f_{1}^{\prime}(x+t)+ \\
& +a(s, x-t) f_{1}(x-t)+b(s, x-t) f_{2}(x-t)+c(s, x-t) f_{1}^{\prime}(x-t) \mid \leqq c c_{5} \cdot t \cdot\|f\|_{1}
\end{aligned}
$$

(where $c_{5}$ is a positive constant). Similarly, it may be derived that there exists a nondecreasing positive function $c_{6}(R)$ so that the indequality

$$
\left\|\mathrm{e}^{A t} N(s) f\right\|_{1} \leqq c_{6}(R) t\|f\|_{1}^{2}
$$

holds for all $t \geqq 0, s \geqq 0$ and $f \in X_{1}$ such that $\|f\|_{1} \leqq R$.

Now, we see that the conditions (v), (vi) and (vii) of Section 1 are fulfilled with an arbitrary $p>1$ in (vi) and $\alpha=1$ in (vii).

Using Theorems 2.1 and 3.1, we see that the uniform exponential stability with respect to the norm

$$
\begin{gathered}
\left.\|u(t, .) \mid\|^{\prime}=\|\left[u(t,)_{0}\right), u_{t}(t, .)\right] \mid \|= \\
=\max _{x \in\langle 0, \pi\rangle}|u(t, x)|+2 \max _{x \in\langle 0, \pi\rangle}\left|u_{t}(t, x)\right|+\max _{x \in\langle 0, \pi\rangle}\left|u_{x}(t, x)\right|+ \\
+\max _{x \in\langle 0, \pi\rangle}\left|u_{t x}(t, x)\right|+\max _{x \in\langle 0, \pi\rangle}\left|u_{x x}(t, x)\right|
\end{gathered}
$$

of the zero solution of the equation

$$
u_{t t}-u_{x x}=a(t, x) u+b(t, x) u_{t}+c(t, x) u_{x}
$$


is a sufficient condition for the uniform exponential stability and the uniform stability at constantly acting disturbances with respect to the norm $|\| \cdot|||$ of the zero solution of the equation (0.1) (hence also for the same properties, but with respect to the norm $\|\left.|\cdot|\right|^{\prime}$, of the zero solution of the equation (4.2.10)). Considering the relation between the zero solution of the equation (4.2.10) and the solution $u_{0}$ of the equation (4.2.1), we may conclude:

Theorem 4.2.1. Let the zero solution of the linear equation (4.2.15) (with one of the boundary conditions (4.2.2), (4.2.5) and (4.2.6)) be uniformly exponentially stable with respect to the norm $\|\cdot \mid\|^{\prime}$. Then the solution $u_{0}$ of the equation (4.2.1) (with the same boundary conditions as in the case of the equation (4.2.15)) is uniformly exponentially stable and uniformly stable at constantly acting disturbances with respect to the norm $\|\cdot\| \|^{\prime}$.

Remark 4.2.1. The same results as in Theorem 4.2.1 could be proved also with respect to the norm

$$
\|u(t, .)\| \|^{\prime \prime}=\left\{\int_{0}^{\pi}\left[u^{2}(t, x)+u_{t}^{2}(t, x)+u_{x}^{2}(t, x)+u_{t x}^{2}(t, x)+u_{x x}^{2}(t, x)\right] \mathrm{d} x\right\}^{1 / 2}
$$

instead of $\|\cdot \mid\|^{\prime}$. Then the magnitude of disturbances could be determined by the norm

$$
\|u(t, .)\|_{0}^{\prime \prime}=\left\{\int_{0}^{\pi}\left[u^{2}(t, x)+u_{x}^{2}(t, x) \mathrm{d} x\right\}^{1 / 2}\right.
$$

(see [12]). We investigate in detail the uniform exponential stability with respect to the norm $\|\cdot\|\|\|^{\prime}$ of the zero solution of several rather more special cases of the linear equation (4.2.15) and then apply the results obtained to the corresponding special cases of the equation (4.2.1) also in [12].

\subsection{THE TIMOSHENKO TYPE EQUATION}

Suppose that $H$ is a Hilbert space with the norm $\|\cdot\|_{H}$ and $L$ is a selfadjoint, strictly positive linear operator in $H$ with the domain of definition $\mathscr{D}(L)$ (the strict positivity of the operator $L$ means that $\varrho=\inf \operatorname{Sp}(L)>0)$. Let $u_{0} \in \bigcap_{i=0}^{4} C^{i}\left(\langle 0,+\infty) ; \mathscr{D}\left(L^{1-i / 4}\right)\right)$
be a solution of the equation

$$
\begin{gathered}
u^{\prime \prime \prime \prime}(t)+\alpha L^{1 / 2} u^{\prime \prime}(t)+\beta L u(t)= \\
=F\left(t, u, L^{1 / 4} u, L^{1 / 2} u, L^{3 / 4} u, u^{\prime}, L^{1 / 4} u^{\prime}, L^{1 / 2} u^{\prime}, u^{\prime \prime}, L^{1 / 4} u^{\prime \prime}, u^{\prime \prime \prime}\right),
\end{gathered}
$$


where $\alpha, \beta$ are real constants and $F$ is a function with values in $H$. The operators $L^{v}$ (for $v=0, \frac{1}{4}, \frac{1}{2}, \frac{3}{4}$ ) may be defined as follows:

$$
L^{v} u=\int_{\varrho}^{+\infty} s^{v} \mathrm{~d} E(s) u,
$$

where $E(s)$ is the spectral resolution of identity corresponding to the operator $L$. Provided $F$ is sufficiently smooth, the problem of the uniform exponential stability or the uniform stability at constantly acting disturbances of the solution $u_{0}$ may be transformed to the same problem concerning the zero solution of the equation of the type

$$
\begin{aligned}
& u^{\prime \prime \prime \prime}+\alpha L^{1 / 2} u^{\prime \prime}+\beta L u=a_{1}(t) u+a_{2}(t) L^{1 / 4} u+a_{3}(t) L^{1 / 2} u+a_{4}(t) L^{3 / 4} u+ \\
& +b_{1}(t) u^{\prime}+b_{2}(t) L^{1 / 4} u^{\prime}+b_{3}(t) L^{1 / 2} u^{\prime}+c_{1}(t) u^{\prime \prime}+c_{2}(t) L^{1 / 4} u^{\prime \prime}+ \\
& +d(t) u^{\prime \prime \prime}+G\left(t, u, L^{1 / 4} u, L^{1 / 2} u, L^{3 / 4} u, u^{\prime}, L^{1 / 4} u^{\prime}, L^{1 / 2} u^{\prime}, u^{\prime \prime}, L^{1 / 4} u^{\prime \prime}, u^{\prime \prime \prime}\right),
\end{aligned}
$$

where $a_{1}(t), a_{2}(t), \ldots, c_{2}(t), d(t)$ are bounded linear operators in $H$ such that

a1) $\left\|a_{1}(t) u_{1}+a_{2}(t) L^{1 / 4} u_{1}+\ldots+d(t) u_{4}\right\|_{H} \leqq c_{5} \sum_{i=1}^{4} \sum_{j=0}^{4-i}\left\|L^{j / 4} u_{i}\right\|_{H}$

(for $t \geqq 0$ and $\left.\left[u_{1}, u_{2}, u_{3}, u_{4}\right] \in \mathscr{D}\left(L^{3 / 4}\right) \times \mathscr{D}\left(L^{1 / 2}\right) \times \mathscr{D}\left(L^{1 / 4}\right) \times H\right)$,

b1) if $\left[u_{1}, u_{2}, u_{3}, u_{4}\right] \in \mathscr{D}(L) \times \mathscr{D}\left(L^{3 / 4}\right) \times \mathscr{D}\left(L^{1 / 2}\right) \times \mathscr{D}\left(L^{1 / 4}\right)$, then

$a_{1}(t) u_{1}+a_{2}(t) L^{1 / 4} u_{1}+\ldots+d(t) u_{4} \in \mathscr{D}\left(L^{1 / 4}\right)$,

c1) $\left\|L^{1 / 4}\left[a_{1}(t) u_{1}+a_{2}(t) L^{1 / 4} u_{1}+\ldots+d(t) u_{4}\right]\right\|_{H} \leqq c_{5} \sum_{i=1}^{4} \sum_{j=1}^{5-i}\left\|L^{j / 4} u_{i}\right\|_{H}$

(for $t \geqq 0$ and $\left.\left[u_{1}, u_{2}, u_{3}, u_{4}\right] \in \mathscr{D}(L) \times \mathscr{D}\left(L^{3 / 4}\right) \times \mathscr{D}\left(L^{1 / 2}\right) \times \mathscr{D}\left(L^{1 / 4}\right)\right)$.

$G\left(t, u, L^{1 / 4} u, L^{1 / 2} u, \ldots, L^{1 / 4} u^{\prime \prime}, u^{\prime \prime \prime}\right)$ is a nonlinear operator in $H$ such that

a2) $\left\|G\left(t, u_{1}, L^{1 / 4} u_{1}, L^{1 / 2} u_{1}, L^{3 / 4} u_{1}, u_{2}, L^{1 / 4} u_{2}, L^{1 / 2} u_{2}, u_{3}, L^{1 / 4} u_{3}, u_{4}\right)\right\|_{H} \leqq$

$\leqq c_{6}(R)\left[\sum_{i=1}^{4} \sum_{j=0}^{4-i}\left\|L^{j / 4} u_{i}\right\|_{H}\right]^{2}$

(for $t \geqq 0$ and $\left[u_{1}, u_{2}, u_{3}, u_{4}\right] \in \mathscr{D}\left(L^{3 / 4}\right) \times \mathscr{D}\left(L^{1 / 2}\right) \times \mathscr{D}\left(L^{1 / 4}\right) \times H$ such that

$$
\left.\sum_{i=1}^{4} \sum_{j=0}^{4-i}\left\|L^{j / 4} u_{i}\right\|_{H} \leqq R\right),
$$

b2) if $\left[u_{1}, u_{2}, u_{3}, u_{4}\right] \in \mathscr{D}(L) \times \mathscr{D}\left(L^{3 / 4}\right) \times \mathscr{D}\left(L^{1 / 2}\right) \times \mathscr{D}\left(L^{1 / 4}\right)$ then

$G\left(t, u_{1}, L^{1 / 4} u_{1}, L^{1 / 2} u_{1}, \ldots, L^{1 / 4} u_{3}, u_{4}\right) \in \mathscr{D}\left(L^{1 / 4}\right)$,

c2)

$\left\|L^{1 / 4} G\left(t, u_{1}, L^{1 / 4} u_{1}, L^{1 / 2} u_{1}, L^{3 / 4} u_{1}, \ldots, L^{1 / 4} u_{3}, u_{4}\right)\right\|_{H} \leqq c_{6}(R)\left[\sum_{i=1}^{4} \sum_{j=0}^{5-i}\left\|\left.L^{j}\right|^{4} u_{i}\right\|_{H}\right]^{2}$

(for $t \geqq 0$ and $\left[u_{1}, u_{2}, u_{3}, u_{4}\right] \in \mathscr{D}(L) \times \mathscr{D}\left(L^{3 / 4}\right) \times \mathscr{D}\left(L^{1 / 2}\right) \times \mathscr{D}\left(L^{1 / 4}\right)$ such that

$$
\left.\sum_{i=1}^{4} \sum_{j=0}^{5-i}\left\|L^{j / 4} u_{i}\right\|_{H} \leqq R\right) \text {. }
$$


Put $X=\mathscr{D}\left(L^{3 / 4}\right) \times \mathscr{D}\left(L^{1 / 2}\right) \times \mathscr{D}\left(L^{1 / 4}\right) \times H$ and define

$$
\|f\|_{0}=\sum_{i=1}^{4} \sum_{j=1}^{4-i}\left\|L^{1 / 4} f_{i}\right\|_{H} \quad\left(\text { for } f=\left[f_{1}, f_{2}, f_{3}, f_{4}\right] \in X\right) .
$$

Since $L^{v}$ (for $v=0, \frac{1}{4}, \frac{1}{2}$ and $\frac{3}{4}$ ) are closed operators in $H$ (see e.g. [18]), $X$ is a Banach space with the norm $\|\cdot\|_{0}$.

Let us define

$$
\begin{aligned}
& A f=\left[f_{2}, f_{3}, f_{4},-\alpha L^{1 / 2} f_{3}-\beta L f_{1}\right]\left(\text { for } f \in \mathscr{D}(L) \times \mathscr{D}\left(L^{3 / 4}\right) \times \mathscr{D}\left(L^{1 / 2}\right) \times \mathscr{D}\left(L^{1 / 4}\right) \equiv\right. \\
& \quad \equiv \mathscr{D}(A)), \\
& B(t) f=\left[0,0,0, a_{1}(t) f_{1}+a_{2}(t) L^{1 / 4} f_{1}+a_{3}(t) L^{1 / 2} f_{1}+\ldots+c_{2}(t) L^{1 / 4} f_{3}+d(t) f_{4}\right], \\
& N(t) f=\left[0,0,0, G\left(t, f_{1}, L^{1 / 4} f_{1}, L^{1 / 2} f_{1}, \ldots, L^{1 / 4} f_{3}, f_{4}\right)\right](\text { for } f \in X) .
\end{aligned}
$$

Then denoting $U=\left[u, u^{\prime}, u^{\prime \prime}, u^{\prime \prime \prime}\right]$, we can rewrite the equation (4.3.2) in the form (0.1):

$$
\frac{\mathrm{d} U}{\mathrm{~d} t}=A U+B(t) U+N(t) U
$$

It may be proved that if

$$
\alpha>0, \quad \beta>0, \quad \alpha^{2}-4 \beta \geqq 0
$$

then the operator $A$ is the generator of a semigroup of operators $\mathrm{e}^{A t}$ of the class $C_{0}$ such that

$$
\begin{gathered}
\mathrm{e}^{A t} f=\left[\sum_{i=1}^{4} \int_{\varrho}^{+\infty} m_{i}(t, s) \mathrm{d} E(s) f_{i}, \sum_{i=1}^{4} \int_{\varrho}^{+\infty} \frac{\partial m_{i}(t, s)}{\partial t} \mathrm{~d} E(s) f_{i},\right. \\
\left.\sum_{i=1}^{4} \int_{\varrho}^{+\infty} \frac{\partial^{2} m_{i}(t, s)}{\partial t^{2}} \mathrm{~d} E(s) f_{i}, \sum_{i=1}^{4} \int_{\varrho}^{+\infty} \frac{\partial^{3} m_{i}(t, s)}{\partial t^{3}} \mathrm{~d} E(s) f_{i}\right]
\end{gathered}
$$

for $f=\left[f_{1}, f_{2}, f_{3}, f_{4}\right] \in X$, where $m_{i}(\cdot, s)(i=1,2,3,4)$ are solutions of an ordinary differential equation

$$
\frac{\partial^{4} m_{i}(t, s)}{\partial t^{4}}+\alpha \sqrt{ } s \frac{\partial^{2} m_{i}(t, s)}{\partial t^{2}}+\beta s m_{i}(t, s)=0
$$

on $\langle 0,+\infty)$, fulfilling initial conditions

$$
\frac{\partial^{j} m_{i}(0, s)}{\partial t^{j}}=\delta_{i, j-1} \quad(\text { for } i=1,2,3,4 ; j=0,1,2,3 ; s \in(\varrho,+\infty)) .
$$

We can find that

$$
m_{1}(t, s)=\frac{\lambda_{2}^{2}}{\lambda_{2}^{2}-\lambda_{1}^{2}} \cos \left(s^{1 / 4} \lambda_{1} t\right)+\frac{\lambda_{1}^{2}}{\lambda_{1}^{2}-\lambda_{2}^{2}} \cos \left(s^{1 / 4} \lambda_{2} t\right),
$$




$$
\begin{aligned}
& m_{2}(t, s)=\frac{\lambda_{2}^{2}}{s^{1 / 4} \lambda_{1}\left(\lambda_{2}^{2}-\lambda_{1}^{2}\right)} \sin \left(s^{1 / 4} \lambda_{1} t\right)+\frac{\lambda_{1}^{2}}{s^{1 / 4} \lambda_{2}\left(\lambda_{1}^{2}-\lambda_{2}^{2}\right)} \sin \left(s^{1 / 4} \lambda_{2} t\right), \\
& m_{3}(t, s)=\frac{1}{s^{1 / 2}\left(\lambda_{2}^{2}-\lambda_{1}^{2}\right)} \cos \left(s^{1 / 4} \lambda_{1} t\right)+\frac{1}{s^{1 / 2}\left(\lambda_{1}^{2}-\lambda_{2}^{2}\right)} \cos \left(s^{1 / 4} \lambda_{2} t\right), \\
& m_{4}(t, s)=\frac{1}{s^{3 / 4} \lambda_{1}\left(\lambda_{2}^{2}-\lambda_{1}^{2}\right)} \sin \left(s^{1 / 4} \lambda_{1} t\right)+\frac{1}{s^{3 / 4} \lambda_{2}\left(\lambda_{1}^{2}-\lambda_{2}^{2}\right)} \sin \left(s^{1 / 4} \lambda_{2} t\right),
\end{aligned}
$$

where $\lambda_{1}=\sqrt{ }\left(\alpha-\sqrt{ }\left(\alpha^{2}-4 \beta\right)\right) / 2, \lambda_{2}=\sqrt{ }\left(\alpha+\sqrt{ }\left(\alpha^{2}-4 \beta\right)\right) / 2$. Thus, it is

$$
\left|\frac{\partial^{j} m_{i}(t, s)}{\partial t^{j}}\right| \leqq \text { const. } s^{(j-i+1) / 4} \quad(\text { for } t \geqq 0, s>\varrho ; i=1,2,3,4 ; j=0,1,2,3) \text {. }
$$

The integral $\int_{\varrho}^{+\infty} s^{(4-i) / 4} \mathrm{~d} E(s) f_{i}(i=1,2,3,4)$ converges for $f_{i} \dot{E} \mathscr{D}\left(L^{(4-i) / 4}\right)$, i.e. also the integral

$$
\int_{\varrho}^{+\infty} \frac{\partial^{j} m_{i}(t, s)}{\partial t^{j}} \mathrm{~d} E(s) f_{i} \quad(\text { for } i=1,2,3,4 ; j=0,1,2,3)
$$

converges and hence all terms in (4.3.4) have sense.

The operators $B(t)$ and $N(t)$ transform $X$ into itself and it holds

$\|B(t) f\|_{0} \leqq c_{5}\|f\|_{0} \quad$ (for $t \geqq 0$ and $f \in X$ ),

$\|A B(t) f\|_{0} \leqq c_{5}\|A f\|_{0} \quad$ (for $t \geqq 0$ and $\left.f \in \mathscr{D}(A)\right)$,

$\|N(t) f\|_{0} \leqq c_{6}(R)\|f\|_{0}^{2} \quad\left(\right.$ for $t \geqq 0$ and $f \in X$ such that $\left.\|f\|_{0} \leqq R\right)$,

$\|A N(t) f\|_{0} \leqq c_{6}(R)\|A f\|_{0}^{2}$ (for $t \geqq 0$ and $f \in \mathscr{D}(A)$ such that $\|A f\|_{0} \leqq R$ ).

Let $X_{1}$ be the space $\mathscr{D}(A)$ with the norm $\|\cdot\|_{1}=\|\cdot\|_{0}+\|A \cdot\|_{0}$. Put $\mathscr{D}\left(\|\cdot\| \|_{0}\right)=X$, $\|\cdot\|_{0}=\|\cdot\|_{0},\|\cdot\|\|=\| \cdot \|_{1}$. Then it may be easily proved that the conditions (v) $-(\mathrm{vii})$ from $\S 1$ are satisfied. For example:

$$
\begin{gathered}
\left\|\mathrm{e}^{A t} N(s) f\right\|=\left\|\mathrm{e}^{A t} N(s) f\right\|_{0}+\left\|A \mathrm{e}^{A t} N(s) f\right\|_{0} \leqq M_{\varrho} \mathrm{e}^{\varrho t}\left(\|N(s) f\|_{0}+\right. \\
\left.+\|A N(s) f\|_{0}\right) \leqq M_{\varrho} \mathrm{e}^{\varrho t} c_{6}(R)\|f\|_{1}^{2}=M_{\varrho} e^{\varrho t} c_{6}(R)\|f\|^{2}
\end{gathered}
$$

and so the inequality (1.5) in the condition (vii) holds.

Now it is seen that we can apply Theorem 2.1 and Theorem 3.1 and, considering the relations between the equations (4.3.2) and (0.1), we conclude that the following theorem holds:

Theorem 4.3.1. Let the zero solution of the linear equation

$$
u^{\prime \prime \prime \prime}+\alpha L^{1 / 2} u^{\prime \prime}+\beta L u=0 \quad\left(\alpha>0, \beta>0, \alpha^{2}-4 \beta>0\right)
$$


be uniformly exponentially stable with respect to the norm

$$
\begin{gathered}
\|u(t)\|^{\prime}=\|u(t)\|_{H}+\left\|L^{1 / 4} u(t)\right\|_{H}+\left\|L^{1 / 2} u(t)\right\|_{H}+\left\|L^{3 / 4} u(t)\right\|_{H}+\|L u(t)\|_{H}+ \\
+\left\|u^{\prime}(t)\right\|_{H}+\left\|L^{1 / 4} u^{\prime}(t)\right\|_{H}+\left\|L^{1 / 2} u^{\prime}(t)\right\|_{H}+\left\|L^{3 / 4} u^{\prime}(t)\right\|_{H}+\left\|u^{\prime \prime}(t)\right\|_{H}+ \\
+\left\|L^{1 / 4} u^{\prime \prime}(t)\right\|_{H}+\left\|L^{1 / 2} u^{\prime \prime}(t)\right\|_{H}+\left\|u^{\prime \prime \prime}(t)\right\|_{H}+\left\|L^{1 / 4} u^{\prime \prime \prime}(t)\right\|_{H} .
\end{gathered}
$$

Then the zero solution of the equation (4.3.2) is uniformly exponentially stable and uniformly stable at constantly acting disturbances with respect to the norm $\|\mid\| \|^{\prime}$. The magnitude of disturbances is supposed to be determined by the norm

$$
\begin{gathered}
\|\| v(t)\left\|_{0}^{\prime}=\right\| v(t)\left\|_{H}+\right\| L^{1 / 4} v(t)\left\|_{H}+\right\| L^{1 / 2} v(t)\left\|_{H}+\right\| L^{3 / 4} v(t)\left\|_{H}+\right\| v^{\prime}(t) \|_{H}+ \\
+\left\|L^{1 / 4} v^{\prime}(t)\right\|_{H}+\left\|L^{1 / 2} v^{\prime}(t)\right\|_{H}+\left\|v^{\prime \prime}(t)\right\|_{H}+\left\|L^{1 / 4} v^{\prime \prime}(t)\right\|_{H}+\left\|v^{\prime \prime \prime}(t)\right\|_{H} .
\end{gathered}
$$

Remark 4.3.1. The exponential stability of a linear Timoshenko type equation

$$
u^{\prime \prime \prime \prime}+a u^{\prime \prime \prime}+\left(b_{1} L^{1 / 2}+b_{2} I\right) u^{\prime \prime}+\left(c_{1} L^{1 / 2}+c_{2} I\right) u^{\prime}+\left(d_{1} L+d_{2} L^{1 / 2}+d_{3} I\right)=0
$$

(where $a, b_{1}, b_{2}, c_{1}, c_{2}, d_{1}, d_{2}, d_{3}$ are real constants) is investigated in detail in the paper [1].

\section{References}

[1] Barták J.: The Lyapunov Stability of the Timoshenko Type Equation. Čas. pro pěst. matematiky 101, 130-139, 1976.

[2] Barták J.: The Lyapunov Stability and Stability at Constantly Acting Disturbances of an Abstract Differential Equation of the Second Order. Czech. Math. Journal 26 (101), 411 to 437, 1976.

[3] Dunford N. - J. T. Schwartz: Linear Operators I. New York-London, 1958.

[4] Filatov A. N.: Averaging Methods in Differential and Integral Equations. Tashkent, FAN 1971 (Russian).

[5] Iooss G.: Théorie non linéaire de la stabilité des écoulements laminaires dans le cas de „l'échange des stabilités“. Arch Rat. Mech. Anal. 40, 166-208, 1971.

[6] Iooss G.: Existence et stabilité de la solution périodique secondaire intervenant dans les problémes d'évolution du type Navier-Stokes. Arch. Rat. Mech. Anal. 47, 301-329, 1972.

[7] Iooss G.: Bifurcation of a Periodic Solution of the Navier-Stokes Equations into an Invariant Torus. Arch. Rat. Mech. Anal. 58, 35-56, 1975.

[8] Joseph D. D. - D. A. Nield: Stability of Bifurcating Time-Periodic and Steady Solutions of Arbitrary Amplitude. Arch. Rat. Mech. Anal. 58, 369-380., 1975.

[9] Judovič V. I.: Appearance of Auto-Oscillations in a Fluid. Prikl. Mat. Mech. 35, 638-655, 1971 (Russian).

[10] Kielhöfer H.: Stability and Semilinear Evolution Equations in Hilbert Space. Arch. Rat. Mech. Anal. 57, 150-165, 1974.

[11] Ladyzhenskaya O. A.: The Mathematical Theory of Viscous Incompressible Flow. Moskva, Nauka 1970 (Russian). 
[12] Neustupa J.: The Uniform Exponential Stability and the Stability at Constantly Acting Disturbances of a Periodic Solution of the Wave Equation. Czech. Math. Journal 26 (101), $388-410,1976$.

[13] Prodi G.: Teoremi di tipo locale per il sistema di Navier-Stokes e stabilitá delle soluzioni stazionarie, Rend. Sem. Mat. Univ. Padova 32, 374-397, 1962.

[14] Sattinger D. H.: The Mathematical Problem of Hydrodynamic Stability. Journal of Math. and Mech. 18, No. 9, 797-817, 1970.

[15] Sattinger D. H.: Topics in Stability and Bifurcation Theory. Lecture Notes in Mathematics, n. 309, Berlin-Heidelberg-New York: Springer 1973.

[16] Sova M.: On the Timoshenko Type Equations. Čas. pro pěst. matematiky 100, 217-254, 1975.

[17] Sova M.: Linear Differential Equations in Banach Spaces. Rozpravy ČSAV, řada mat. a př́r. věd, 85, 6, Academia Praha 1975.

[18] Yosida K.: Functional Analysis. Berlin—Göttingen-Heidelberg, Springer 1965.

Author's address: Suchbátarova 4, 16607 Praha 6, ČSSR (Katedra aplikované matematiky a výpočtové techniky, Strojní fakulta ČVUT). 